ARTICLE

Translational Therapeutics

\title{
Dasatinib sensitises triple negative breast cancer cells to chemotherapy by targeting breast cancer stem cells
}

\author{
Jun Tian ${ }^{1}$, Fatmah Al Raffa ${ }^{1}$, Meiou Dai ${ }^{1}$, Alaa Moamer ${ }^{1}$, Baharak Khadang ${ }^{2}$, Ibrahim Y. Hachim ${ }^{1}$, Khldoun Bakdounes ${ }^{2,3}$, Suhad Ali ${ }^{1}$, \\ Bertrand Jean-Claude ${ }^{1}$ and Jean-Jacques Lebrun ${ }^{1}$
}

BACKGROUND: Patients with triple negative breast cancer (TNBC) exhibit poor prognosis and are at high risk of tumour relapse, due to the resistance to chemotherapy. These aggressive phenotypes are in part attributed to the presence of breast cancer stem cells (BCSCs). Therefore, targeting BCSCs is a priority to overcoming chemotherapy failure in TNBCs.

METHODS: We generated paclitaxel (pac)-resistant TNBC cells which displayed higher sphere forming potential and percentage of BCSC subpopulations compared to the parental cells. A screen with various kinase inhibitors revealed dasatinib, a Src kinase family inhibitor, as a potent suppressor of BCSC expansion/sphere formation in pac-resistant TNBC cells.

RESULTS: We found dasatinib to block pac-induced BCSC enrichment and Src activation in both parental and pac-resistant TNBC cells. Interestingly, dasatinib induced an epithelial differentiation of the pac-resistant mesenchymal cells, resulting in their enhanced sensitivity to paclitaxel. The combination treatment of dasatinib and paclitaxel not only decreased the BCSCs numbers and their sphere forming capacity but also synergistically reduced cell viability of pac-resistant cells. Preclinical models of breast cancer further demonstrated the efficiency of the dasatinib/paclitaxel combination treatment in inhibiting tumour growth. CONCLUSIONS: Dasatinib is a promising anti-BCSC drug that could be used in combination with paclitaxel to overcome chemoresistance in TNBC.

British Journal of Cancer (2018) 119:1495-1507; https://doi.org/10.1038/s41416-018-0287-3

\section{BACKGROUND}

Triple negative breast cancers (TNBCs) exhibit aggressive features and poor patient outcomes. Due to the lack expression of oestrogen receptor (ER), progesterone receptor (PR) and human epidermal growth factor receptor 2 (HER2), TNBC is not amenable to anti-hormone therapy or anti-HER2 targeted therapy, thereby dramatically increasing the death toll by this type of tumour. ${ }^{1}$ Although showing an initial response to cytotoxic chemotherapeutic agents, the majority of TNBC patients still exhibit poor prognosis and are at high risk of tumour relapse and metastasis because of intrinsic and acquired drug resistance. ${ }^{2}$

The ineffectiveness of chemotherapy treatments has been attributed to the presence of cancer stem-like cells (CSCs), a subpopulation of cancer cells that possesses the ability to selfrenew and to generate heterogeneous tumours., ${ }^{3,4}$ CSCs are relatively quiescent and intrinsically resistant to chemotherapy. TNBCs are enriched in breast cancer stem cells (BCSCs) that are characterised by expression of cell surface marker CD24 low CD44 ${ }^{\text {high }}$ and enhanced ALDH activity. ${ }^{5,6}$ Hence, new therapeutic options to overcome chemotherapy resistance and induce longlasting clinical responses for TNBCs are in need. As such, eliminating drug-resistant BCSCs becomes a priority for the development of novel anti-TNBC therapies.

Other therapeutic strategies aim at inducing terminal differentiation of CSCs, thereby increasing their sensitivity to cytotoxic chemotherapy. For instance, induced differentiation of colorectal cancer stem cells could sensitise them to chemotherapy both in vitro and in vivo. ${ }^{7,8}$ Similarly, in vitro differentiation of stemlike glioma cells by all-trans-retinoic acid could contribute to chemosensitisation. ${ }^{9}$ Although differentiation of CSCs has proved efficient to achieve better responses to chemotherapy in colon cancer and glioblastoma, this remains unclear in the context of breast cancer. In this study, we focused on identifying agents that could either eliminate or induce differentiation of pac-resistant BCSCs and on assessing the efficacy of these agents in improving responses to chemotherapy. Indeed, combination of novel BCSCtargeting drugs with cytotoxic chemotherapy targeting differentiated breast cancer could further lead to successful tumour depletion and achieve a better clinical outcome for patients with chemotherapy-resistant TNBC.

Src is a non-receptor tyrosine kinase that mediates many cellular events, such as cell proliferation, growth and survival. ${ }^{10}$ Aberrant activation of Src signalling has been observed in various cancers, including those of the breast. ${ }^{10-13}$ Src has also been implied in the regulation of breast cancer stemness and metastasis. $^{14,15}$ Dasatinib is the first FDA-approved SFK/ABL dual inhibitor for the treatment of chronic myeloid leukaemia and Philadelphia chromosome-positive acute lymphoblastic leukaemia. ${ }^{16}$ Preclinical studies also demonstrated that dasatinib could inhibit growth of breast cancer cells ${ }^{17}$ and is associated with a

\footnotetext{
${ }^{1}$ Department of Medicine, McGill University Health Center, Cancer Research Program, Montreal, Quebec H4A 3J1, Canada; ${ }^{2}$ Department of Pathology, McGill University Health Center, Montreal, Quebec H4A 3J1, Canada and ${ }^{3}$ Department of Pathology, St. Mary's Hospital, Montreal, Quebec H3T 1M5, Canada Correspondence: J-J. Lebrun (JJ.lebrun@mcgill.ca)
} 
decreased percentage of ALDH+ BCSCs in basal-b subtype of breast cancer. ${ }^{18}$ As BCSCs are the driving cells for chemotherapy resistance, we evaluated the role of dasatinib in targeting BCSCs and its ability to overcome chemotherapy resistance. We found that pac-resistant cells displayed higher BCSC sphere forming capacity and higher active Src kinase level compared to parental cells and showed that dasatinib treatment completely abolished these effects. We also found dasatinib to block pac-induced Src activation as well as BCSC sphere formation capacity and expansion in parental TNBC cells. Moreover, we demonstrated that the combination of dasatinib and paclitaxel synergistically reduced cell viability of pac-resistant cells in vitro and significantly inhibited breast tumour growth in vivo, suggesting the dasatinib/ paclitaxel combination treatment as a potential therapy for overcoming chemotherapy resistance in TNBC patients.

\section{MATERIALS AND METHODS}

All experimental protocols and procedures were performed in accordance to McGill University regulations. All experimental protocols and procedures were approved by McGill University.

\section{Drugs}

LY294002, SB431542, Gefitinib, Dasatinib, Rapamycin and palbociclib were purchased from Sigma-Aldrich. JNK inhibitor II and PD98059 were bought from Calbiochem.

Cell culture

SUM159 cells were grown in F-12 HAM'S serum, with 5\% foetal bovine serum (FBS), $5 \mu \mathrm{g} / \mathrm{ml}$ insulin and $1 \mu \mathrm{g} / \mathrm{ml}$ hydrocortisone. Paclitaxel-resistant SUM159 cells were generated by exposure to six to eight cycles of treatments with $10 \mathrm{nM}$ paclitaxel.

\section{Cell viability assay}

Cells were seeded into 96 -well plates $(5,000$ cells/well) and treated with a dose range of drugs. After $48 \mathrm{~h}$, cells were incubated with PrestoBlue ${ }^{\mathrm{TM}}$ Cell Viability Reagent (Thermo Fisher Scientific) for $1 \mathrm{~h}$ at $37^{\circ} \mathrm{C}$. Plates were then subjected to fluorescence reading (excitation: $535 \mathrm{~nm}$, emission: $615 \mathrm{~nm}$ ) using a microplate reader. IC50 drug values were determined using GraphPad Prism 6.0 (GraphPadSoftware, Inc., San Diego, CA) and the combination index $(\mathrm{Cl})$ was determined with the CompuSyn 1.0 software.

\section{Tumoursphere assay}

Cells were seeded in ultra-low-attachment 24-well plate $(10,000$ / well) and cultured in serum-free HAM'S F12 medium supplemented with $10 \mathrm{ng} / \mathrm{ml} \mathrm{EGF,} 10 \mathrm{ng} / \mathrm{ml} \mathrm{bFGF}$ and $1 \times B 27$ (Invitrogen). For treatment, each inhibitor was added at IC50 value. After 7 days, numbers of tumourspheres ( $>60 \mu \mathrm{m}$ diameter) were counted using ImageJ software and sphere-forming efficiency (SFE) was calculated using the following equation: SFE $(\%)=$ (no. of spheres) $/$ (no. of cells plated) $\times 100 \%$.

\section{Flow cytometry}

Monolayer cells were dissociated with trypsin and blocked in prechilled $2 \%$ FBS in PBS for $30 \mathrm{~min}$ at $4{ }^{\circ} \mathrm{C}$. Cells were filtered through $70 \mu \mathrm{m}$ cell strainer and counted. For each sample, 250,000 cells were co-stained with PE-conjugated anti-CD24 and APCconjugated anti-CD44 or stained with APC-conjugated anti-MUC1 antibodies (BD Bioscience, San Diego, USA) for $30 \mathrm{~min}$ on ice. As negative and positive controls, we used non-stained and singlestained cells, respectively. Cells were analysed using Accuri C6 flow cytometer (BD Biosciences) and Flowjo software (Tree Star Inc.).

ALDEFLUOR $^{\text {TM }}$ Kit (Stemcell Technologies) was used to analyse Aldehyde dehydrogenase (ALDH) enzyme activity, according to the manufacturer's protocol. A total of $10^{6}$ cells were incubated with 5 ul ALDH substrate (Bodipy-aminoacetaldehyde) for $45 \mathrm{~min}$ at $37^{\circ} \mathrm{C}$. For the negative control, an aliquot of each sample was incubated with diethylaminobenzaldehyde (DEAB). Analysis were performed using Accuri C6 flow cytometer.

Real-time PCR

Real-time PCR analysis of CDH2, FN1, SNAI1, ZEB1, TP63, ACTA2 and MUC1 were performed using SsoFastTM EvaGreen ${ }^{\circledR}$ Supermix (Bio-Rad) as described previously. ${ }^{19}$ Primer sequences of $\mathrm{CDH} 2$, FN1, SNAI1, TP63, MUC1, ZEB1, ACTA2 and GAPDH were the same as previously used. ${ }^{19,20}$

Western blot

Western blot assay was performed as previously described. ${ }^{19,20}$

3D Matrigel cell culture

A total of 4,000 SUM159-P cells in $100 \mu \mathrm{L}$ growth medium were seeded into poly-D-lysine coated 8-well culture slides coated with $100 \mu \mathrm{L}$ growth factor reduced Matrige ${ }^{\circledR}$. Cells were maintained in growth medium with $5 \%$ Matrigel ${ }^{\circledR}$ for $48 \mathrm{~h}$ for colonies outgrowth. The morphology of the colonies was evaluated after $72 \mathrm{~h}$, following dasatinib treatment. Formed colonies were fixed with $4 \%$ paraformaldehyde, permeabilised using $0.3 \%$ Triton and stained with Alexa Fluor ${ }^{\circledR} 488$ anti-F-actin antibody (Invitrogen), overnight at $4{ }^{\circ} \mathrm{C}$, followed by $4^{\prime}, 6$-diamidino-2-phenylindole (DAPI) staining. Slides were mounted with SlowFade ${ }^{\circledR}$ Gold antifade reagent. Confocal analysis was conducted using a Zeiss LSM 510 Meta Axiovert confocal microscope with $63 \times$ objective.

In vivo studies

All animal experiments were conducted in accordance with protocols approved by the McGill University Health Center. $1 \times$ $10^{6}$ SUM 159 and SUM159-P cells were implanted in the mammary gland of NOD SCID IL2gammaR knock out (NSG) mice. One week after, mice transplanted with SUM159 cells were divided into two groups (6 mice/group) and treated with (i) vehicle $(20 \%$ cremophor $+20 \%$ ethanol $+60 \%$ dextrose, $10 \% \mathrm{w} / \mathrm{v}$ ), (ii) paclitaxel ( $20 \mathrm{mg} / \mathrm{kg}$, once a week). Mice transplanted with SUM159-P cells were randomised into four groups (6 mice/group) and treated with (i) vehicle $(20 \%$ cremophor $+20 \%$ ethanol $+60 \%$ dextrose, $10 \% \mathrm{w} / \mathrm{v})$, (ii) paclitaxel $(20 \mathrm{mg} / \mathrm{kg}$, once a week), (iii) dasatinib $(10 \mathrm{mg} / \mathrm{kg}$, daily), (iv) paclitaxel + dasatinib. Tumour volume were measured using a caliper and mice were weighted every 3 days and determined according to the formula: $\left(L \wedge 2^{*} W\right) / 2$. Upon tumours reaching $700-1100 \mathrm{~mm}^{3}$ in volume, mice were sacrificed and tumours collected for further analyses.

\section{Dissociation of breast tumour xenograft}

$1 \times 10^{6}$ SUM159 and SUM159-P cells were transplanted in the contralateral mammary glands of NSG mice model. Upon reaching $800 \mathrm{~mm}^{2}$ in size, primary mammary tumours were collected, crosscut into small pieces and minced using scalpels. Each tumour tissue was digested using $5 \mathrm{ml}$ collagenase (nine parts of DMEM/ F12 medium mixed with one part of $10 \mathrm{X}$ collagenase/hyaluronidase) and incubated at $37^{\circ} \mathrm{C}$ for $2 \mathrm{~h}$. Cells were centrifuged and incubated with $1 \mathrm{ml}$ Trypsin/EDTA for $5 \mathrm{~min}$. Digestion was stopped by adding $10 \mathrm{ml} 2 \%$ FBS/HBSS. Cells were filtered through 40 uM cell strainers, centrifuged, washed in $2 \% \mathrm{FBS} /$ HBSS, counted and subjected to flow cytometry analysis.

\section{Immunohistochemistry staining and scoring}

Primary tumours were fixed in 10\% formalin, embedded and sectioned. Lab Vision ${ }^{\text {TM }}$ UltraVision ${ }^{\text {TM }}$ LP Detection kit (Thermo Fisher Scientific) was used to perform immunohistochemistry staining. Tumour slides were deparaffinised and rehydrated through a descending series of alcohol then washed four times with buffer. Antigen retrieval was induced through incubation in $10 \mathrm{mM}$ citrate buffer $(\mathrm{pH} 6.0)$ at $90^{\circ} \mathrm{C}$ for $20 \mathrm{~min}$. To reduce nonspecific background, slides were incubated in hydrogen 
peroxide Block for $10 \mathrm{~min}$ and Ultra $\mathrm{V}$ Block for another $5 \mathrm{~min}$. Slides were washed and stained with rabbit polyclonal anti-CD44 antibody (Abcam) at room temperature for $1 \mathrm{~h}$. Slides were incubated in HRP Polymer at room temperature for another 15 min. A mixture of DAB Plus Chromogen and DAB Plus Substrate was applied for detection. Slides were counterstained with hematoxylin and covered using mounting medium.

Mean percentage of IHC staining based on a visual semiquantitative scoring was performed by two pathologists. Membranous and cytoplasmic staining patterns were observed in the viable tumour cells; however, the average percentage of membranous staining was higher than cytoplasmic one. Necrotic and apoptotic parts were excluded. Staining intensity of both the membranes and cytoplasm was assessed separately according to a graded scoring system of 1 (faint to weak, at $\times 40$ magnification), 2 (weak to moderate at $\times 40$ ), 3 (moderate at $\times 20$ ) and 4 (strong at $\times 4)$. The total percentage of both membranous and cytoplasmic staining was used for the assessment of CD44 protein expression in tumour cells.

\section{Statistical analyses}

Student's $t$-test or one-way ANOVA was used to evaluate significance between groups. At least three independent experiments were performed and $P<0.05$ was considered significant.

\section{RESULTS}

Paclitaxel resistance increases the breast cancer stem cell content SUM159PT (hereafter referred to as SUM159), a metastatic human breast cancer cell line derived from a patient with anaplastic breast carcinoma was used in our study, as this cell line is initially sensitive to paclitaxel and has been shown to contain functional cancer stem cell populations. ${ }^{21,22}$ We generated pac-resistant SUM159 cells (SUM159-P) from parental SUM159, using 6-8 cycles of paclitaxel $(10 \mathrm{nM})$ treatment. Each cycle consisted of two days' drug treatment and two days' recovery by culturing cells in drugfree media. Cytotoxic effects of paclitaxel on SUM159 and SUM159-P cells were compared by performing PrestoBlue cell viability assay. As shown in Fig. 1a, SUM159 cells are sensitive to paclitaxel with an IC50 value of $3 \mathrm{nM}$, whereas SUM159-P cells are highly resistant to paclitaxel with an IC50 value of $50 \mathrm{nM}$ (17-fold higher than the parental cells). No change was observed in cell morphology between the parental and pac-resistant cells (Fig. 1b). Recent studies indicated that chemotherapy-treated breast cancer patients displayed increased percentage of BCSCs. ${ }^{23}$ We analysed breast cancer tissue samples from Korde dataset of Oncomine database (www.oncomine.com) and found that paclitaxel-related taxane (docetaxel) treatment of breast cancer led to an increase in stemness/differentiation markers (ALDH1A3 and CD44) (Fig. 1c), while expression of luminal differentiation markers (MUC1 and EpCAM) were decreased (Fig. 1d). We then investigated whether chemotherapy resistance was associated with an increase in BCSCs in SUM159-P cells, using in vitro tumoursphere formation assay, a standard method for assessing CSC numbers. This assay measures the capacity of cells to form three-dimensional spheres in suspension cultures and reflects their ability to self-renew. ${ }^{24}$ As shown in Fig. 1e and f, SUM159-P cells displayed higher sphere forming potential as reflected by the increased SFE compared to the parental SUM159 cells. We then measured ALDH activity and stem cell markers CD24 and CD44 levels in SUM159 and SUM159$P$ cells. We found SUM159-P cells to exhibit significantly higher percentage of $\mathrm{ALDH}+(10.3 \%)$ and $\mathrm{CD} 24^{\text {low }} / \mathrm{CD} 44^{\text {high }}(33.9 \%)$ BCSCs compared to SUM 159 cells $(7.5 \%$ and $24.0 \%$, respectively; Fig. $1 \mathrm{~g}-\mathrm{j}$ ). Moreover, we compared the IC50 value for paclitaxel in both CD24 $4^{\text {low }} / \mathrm{CD} 44^{\text {high }}$ BCSCs and CD24+CD44+non-BCSCs isolated from SUM159 cells and found that the BCSC population is more resistant to paclitaxel treatment with a higher value of IC50 compared to non-BCSCs (Fig. S1). Together, these results indicate that chemotherapy resistance of SUM159-P cells is associated with higher amount of BCSCs and increased sphere forming ability.

To further extend these findings in vivo, we performed xenograft fat pad transplantation of SUM159 and SUM159-P cells in the contralateral mammary glands of NSG mice. ${ }^{25}$ Upon reaching $800 \mathrm{~mm}^{2}$ in size, primary mammary tumours were harvested, dissociated into single cells, and assessed for CD24 low $/ C D 44^{\text {high }}$ BCSC population, using flow cytometry. The BCSC population was markedly increased in SUM159-P derived tumours $(14.8 \%)$ compared with the parental SUM159 tumours $(5.6 \%)$ (Fig. $1 \mathrm{k}, \mathrm{I})$, indicating that paclitaxel-resistant breast cancer cells exhibit enhanced stemness properties, both in vitro and in vivo.

Dasatinib potently eliminates pac-resistant BCSCs

To determine whether pac-resistant TNBC cells are therapeutically treatable, we performed a drug screen with a library of kinasetargeting agents (Table 1). All selected targets are activated in TNBCs and critical for regulating the tumourigenesis processes, thus representing potential therapeutic targets for TNBC. ${ }^{20,26-30}$ Importantly, these kinases have been shown to promote cancer stemness in various models of solid tumours. ${ }^{15,19,20,31-34}$ IC50 doses were determined by cell viability assay in bulk SUM159-P cells (Fig. S2A). We then tested these inhibitors for their ability to modulate BCSC sphere forming capacity, using tumoursphere formation assays. As shown in Fig. 2a, three inhibitors (SB431542, JNK inhibitor II, PD98059) showed moderate effects in decreasing the number of tumourspheres in SUM159-P cells ( 40\% reduction); while another four compounds (LY294002, Gefitinib, Rapamycin, Palbociclib) showed stronger effects (50-80\% reduction). Interestingly, dasatinib reduced tumoursphere-forming efficiency by more than $90 \%$ and inhibited BCSC sphere forming capacity to a greater degree than any other inhibitors. We next assessed the effects of the different inhibitors on the BCSC CD24 $4^{\text {low }} / C D 44^{\text {high }}$ sub-population, using flow cytometry. As shown in Fig. S2B and Fig. 2b, LY294002, SB431542 and Rapamycin had mild to moderate effects on eliminating CD24 $4^{\text {low }} / C D 44^{\text {high }}$ BCSC sub-population ( $35 \%$ reduction), while treatment with Gefitinib, PD98059 and palbociclib showed stronger effects and decreased the proportion of this BCSC subpopulation by $52 \%, 53$ and $68 \%$, respectively. Notably, the percentage of $\mathrm{CD} 24^{\text {low }} / \mathrm{CD} 44^{\text {high }}$ subpopulation was reduced by 95 and $87 \%$ after treatment with dasatinib and JNK inhibitor II, respectively. Altogether, dasatinib was the only inhibitor that could potently prevented BCSC sphere formation and significantly reduced the CD24 low $/ C D 44^{\text {high }}$ BCSC subpopulation. To further confirm the effects of dasatinib on BCSC properties, we assessed its effects on another BCSC sub-population, namely ALDH+ cells. As shown in Fig. 2c, dasatinib treatment resulted in a strong and significant threefold decrease in the proportion of ALDH+ cells, further demonstrating the efficacy of dasatinib in reducing BCSC populations in chemotherapy-resistant breast cancer cells.

Dasatinib suppresses BCSCs through inhibition of paclitaxelinduced Src kinase activation

Having shown that dasatinib drastically inhibited BCSC sphere forming capacity and expansion in pac-resistant cells, we next investigated the underlying mechanisms by examining the phosphorylation status of Src kinase, the main target for dasatinib. Western blot analysis showed that SUM159-P cells exhibit higher level of phospho-Src (Tyr416), compared to parental SUM159 cells, and this was not affected by further addition of paclitaxel (Fig. 3a). Given that SUM159-P cells also contain higher proportion of BCSCs than SUM159 cells, these data suggest a correlation between Src activation and breast cancer stemness. Furthermore, dasatinib treatment completely abolished Src activation in SUM159-P cells without affecting the basal expression of SrC protein (Fig. 3a). Thus, dasatinib-mediated suppression of BCSC 


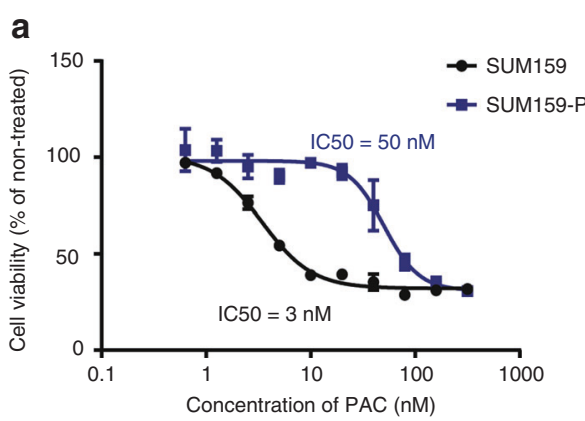

d
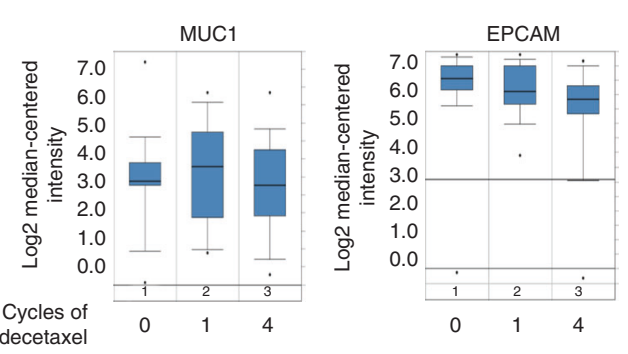

C

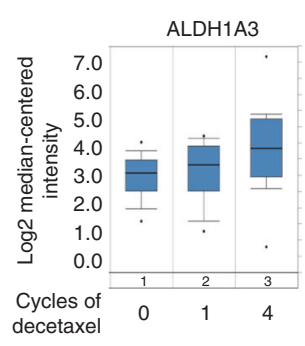

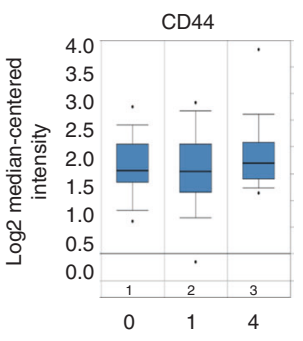

f g

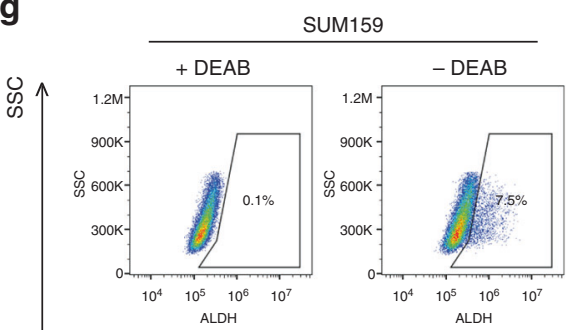

e $\quad$ SUM159

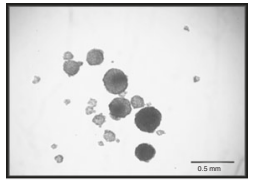

SUM159-P

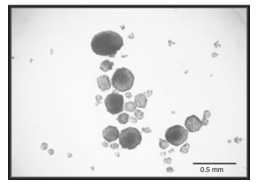

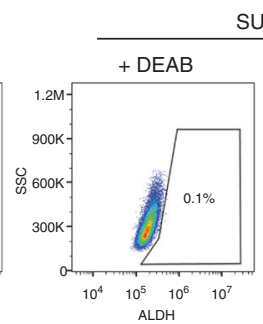

SUM159-P

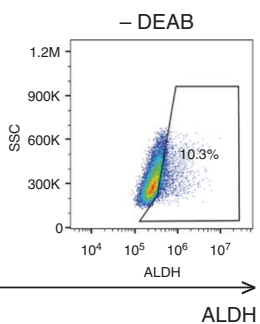

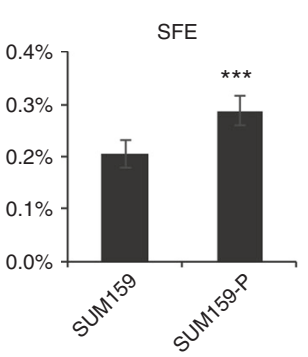

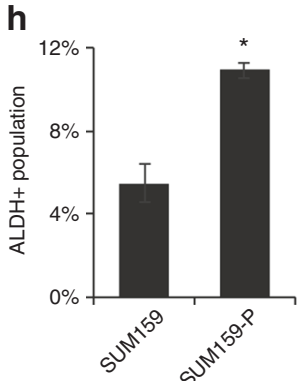

i

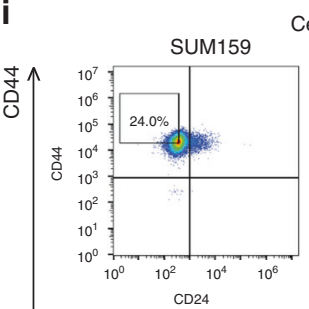

Cell line

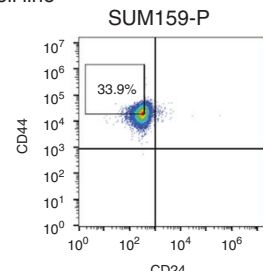

$\mathbf{k}$
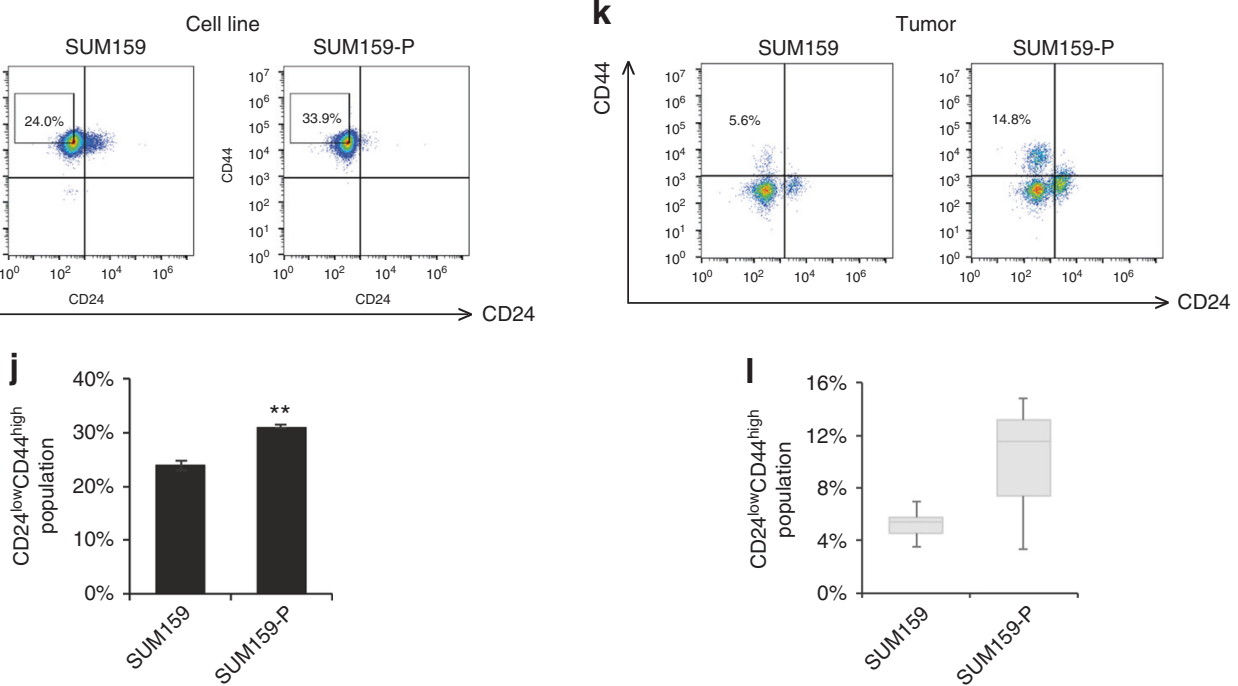

$\rightarrow \mathrm{CD} 24$

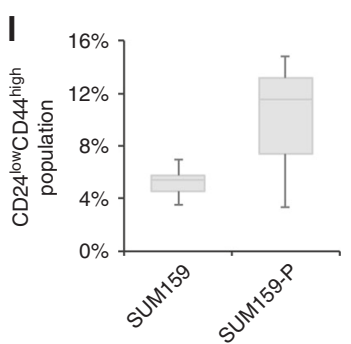

Fig. 1 Paclitaxel resistance is related to stem-like properties. a Cell viability inhibition by different doses of paclitaxel in SUM159 and paclitaxel-resistant SUM159 cells (SUM159-P). The IC50 values of paclitaxel after $48 \mathrm{~h}$ of treatment were determined in both cell lines. $\mathbf{b}$ Phasecontrast microscopic images showed cell morphology of SUM159 and SUM159-P cells. c, d mRNA expression levels of ALDH1A3, CD44, MUC1 and EPCAM in breast cancer patients from the Korde dataset from Oncomine (www.oncomine.com) $(n=21,18,21$, at 0-, 1-, 4-cycle of docetaxel, respectively). e, $\mathbf{f}$ Representative images of SUM159- and SUM159-P-derived tumourspheres. The number of tumourspheres ( $>60 \mu \mathrm{m}$ diameter) was counted and sphere forming efficiency (SFE) was calculated. $\mathbf{g}, \mathbf{h}$ Flow cytometry analysis of ALDH+ BCSCs in SUM159 and SUM159-P cells. DEAB, a specific ALDH inhibitor, was used as a control to determine the ALDH activity. The percentage of ALDH+ populations is graphed. $\mathbf{i}$, j Flow cytometry analysis of CD24 ${ }^{\text {low }}$ CD $44^{\text {high }}$ BCSCs in SUM159 and SUM159-P cells. CD24 ${ }^{\text {low }}$ CD44 $4^{\text {high }}$ population was gated based on high $50 \%$ of CD44+ population and low $50 \%$ of CD24-population. The percentage of CD24 ${ }^{\text {low }}$ CD44 ${ }^{\text {high }}$ populations is indicated. $\mathbf{k}$, I the percentage of CD24 ${ }^{\text {low }} \mathrm{CD} 44^{\text {high }}$ population in cells dissociated from SUM159 and SUM159-P derived tumours. Data are graphed as box and whisker plot, $n=3$ 
Table 1. Inhibitors in the drug screen and their targets

\begin{tabular}{ll}
\hline Inhibitor & Major target(s) \\
\hline LY294002 & PI3K/AKT \\
SB431542 & TGF $\beta$ R1 \\
Gefitinib & EGFR \\
Dasatinib & BCR-Abl, Src \\
Rapamycin & mTOR \\
JNK inhibitor & JNK \\
PD98059 & MEK \\
Palbociclib & CDK4/6
\end{tabular}

stemness in SUM159-P cells could originate from inhibition of pacinduced Src activation resulting from long-term paclitaxel treatments.

BCSCs are intrinsically resistant to conventional treatment and can survive through chemotherapy, giving rise to a population of drug-resistant cancer cells. We hypothesised that short-term treatment with paclitaxel could eliminate the bulk tumour cells and select for the preferential outgrowth of drug-resistant BCSCs. Parental SUM159 cells were treated or not with $10 \mathrm{nM}$ paclitaxel for 4 days, allowed for recover in drug-free media for 4 days and subjected to tumoursphere assay. As shown in Fig. 3b-f, although paclitaxel treatment decreased total cell number of SUM159 cells, it significantly increased the number of tumoursphere forming cells, indicative of an increased BCSC sphere forming activity. Short-term exposure of SUM159 cells to paclitaxel also increased the percentage of ALDH + BCSC population from $8.6 \%$ to $12.7 \%$ (Fig. $3 c, d$ ) and did not significantly change the percentage of CD24 ${ }^{\text {low }} /$ CD $44^{\text {high }}$ BCSC subpopulation (Fig. S3), demonstrating the specific expansion of ALDH+ BCSCs following short term chemotherapy treatment. To address the role and involvement of Src, cells were then treated with dasatinib. While paclitaxel increased phospho-Src levels (Tyr416) in SUM159 cells, dasatinib completely abolished both basal and pac-induced Src phosphorylation (Fig. 3e). Moreover, dasatinib not only markedly reduced the tumoursphere formation and the percentage of ALDH+ cells in parental SUM159 cells, but also blocked pac-induced breast cancer stemness (Fig. 3b-d). Together, these results suggest that pac-increased BCSC sphere formation and expansion are mediated at least in part through Src kinase activity. Besides targeting pac-induced BCSCs enrichment, dasatinib treatment also enhanced the cytotoxic effect of paclitaxel on SUM159 bulk cells (Fig. 3f), suggesting the depletion of both CSCs and non-CSCs after concomitant treatment with dasatinib and paclitaxel.

Dasatinib induces mesenchymal-epithelial transition and differentiation of chemo-resistant cells

The enrichment of cancer stem cells has been shown to correlate with the induction of epithelial-mesenchymal transition (EMT) in breast cancer cells. ${ }^{35}$ Having found that dasatinib prevented BCSC sphere forming capacity and expansion in pac-resistant cells, we sought to investigate whether dasatinib could disrupt the mesenchymal phenotype of these cells. Interestingly, the morphology of SUM159-P cells changed from spindle-like cell shape into cohesive clusters with round cellular morphology after 4 days' treatment with dasatinib, suggesting a mesenchymal-epithelial transition (MET) (Fig. 4a). In accordance with these morphological changes, dasatinib led to decreased mRNA expression of mesenchymal markers, including $\mathrm{N}$-cadherin $(\mathrm{CDH} 2)$, Fibronectin (FN1), Snail (SNAI1), ZEB1 and basal markers TP63, alpha smooth muscle actin (ACTA2) in SUM159-P cells (Fig. 4b). Furthermore, when examined at the protein level, dasatinib treatment led to increased epithelial marker (E-cadherin) expression and decreased expression of the two mesenchymal makers (Fibronectin, Snail)
(Fig. 4c), further indicating that dasatinib contributes to the differentiation of chemo-resistant breast cancer cells by inducing MET.

EMT is a mechanism by which differentiated tumour cells transit to stem-like cancer cells. ${ }^{35}$ Having found dasatinib to induce MET in SUM159-P cells, we examined its effect on phenotypic differentiation. We found increased expression of the luminal differentiation marker MUC1 after dasatinib treatment (Fig. 4d) as well as a fourfold increase in the MUC1+ cell population (Fig. $4 \mathrm{e}$, f). Given that dasatinib suppressed BCSC properties and altered the phenotype of chemo-resistant cells to a more differentiated epithelial cell type in 2D culture, this prompted us to examine whether dasatinib treatment could also lead to the formation of acinar-like structure of SUM159-P cells in three-dimensional (3D) culture. For this, we performed a 3D cell culture on Matrigel to study the patterns of epithelial differentiation in vitro. Indeed, this 3D culture better represents morphology and tumour growth in vivo compared to monolayer cell cultures as it mimics cell-cell and cell-matrix interactions in the tumour microenvironment. ${ }^{36,37}$ In this model, more differentiated cancer cells form acinar-like structures, round spheroids with hollow lumen inside. The more invasive cancer cells, however, form large, poorly differentiated spheroids, or aggressively invading structures. ${ }^{38,39}$ Briefly, SUM159-P cells were cultured in Matrigel for 5 days in the presence or absence of dasatinib and then subjected to immunofluorescence staining of F-actin and Dapi. F-actin staining was performed to better visualise the cell margins. Interestingly, SUM159-P cells formed invasive branching morphogenesis, characterised by the migration of cells through the surrounding Matrigel. In contrast, dasatinib treatment resulted in the formation of round, acinar-like structures characterised by smoothening of the outer layer and development of a central lumen (Fig. 4g). Taken together, these findings indicate that inhibition of Src activity by dasatinib resulted in phenotypic differentiation of chemo-resistant cancer cells in both $2 \mathrm{D}$ and 3D cultures.

\section{Dasatinib-induced differentiation sensitises BCSCs to} chemotherapy

Differentiated cancer cells are more sensitive to conventional chemotherapy than cancer stem cells. Indeed, when analysing 508 breast cancer tissue samples (169 sensitive patients and 339 insensitive patients in Booser dataset from 'R2: Genomics Analysis and Visualisation Platform (http://r2.amc.nl)'), we found a positive correlation between chemotherapy sensitivity and high expression levels of differentiation markers (CD24, EpCAM) and luminal markers (E-cadherin, KRT8, KRT18, MUC1) (Fig. 5a). Having shown that dasatinib induced epithelial differentiation in BCSC-enriched SUM159-P cells, we hypothesised that dasatinib treatment could sensitise the chemo-resistant BCSCs to paclitaxel. To test this hypothesis, SUM159-P cells were treated with DMSO, dasatinib, paclitaxel alone or in combination and subjected to tumoursphere formation assay. Parental SUM159 cells were used as an experimental control. SUM159-P cells exhibited higher numbers of tumoursphere compared to parental SUM159 cells, confirming a higher sphere forming capacity of BCSCs in SUM159-P. Paclitaxel alone didn't affect the tumoursphere formation while dasatinib significantly decreased the BCSC sphere forming ability in SUM159-P cells, as indicated by the significantly decreased sphere forming efficiency (SFE) (Fig. 5b, c). Notably, a combination treatment of dasatinib and paclitaxel showed even stronger effect than dasatinib alone and eliminated nearly all the tumoursphereforming cells (Fig. 5b, c). Thus, these results suggest that dasatinib treatment could sensitise chemo-resistant BCSCs to paclitaxel.

We further evaluated the effects of dasatinib and paclitaxel on CD24 low $/ C D 44^{\text {high }}$ and ALDH + BCSC subpopulations. As shown in Fig. $5 \mathrm{~d}-\mathrm{g}$, we found that paclitaxel alone did not reduce CD24 $4^{\text {low }} /$ CD44 $4^{\text {high }}$ nor ALDH+ BCSC subpopulations, compared to the DMSO treatment. Dasatinib, on the other hand, decreased the 
a
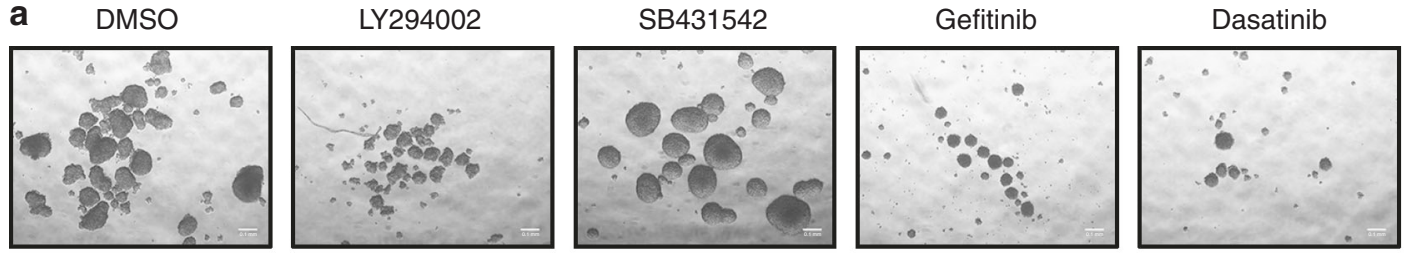

SUM159-P

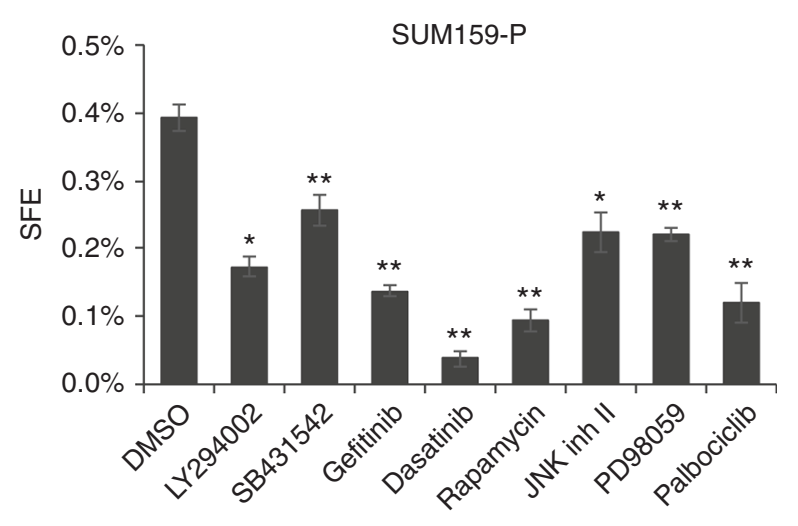

Palbociclib
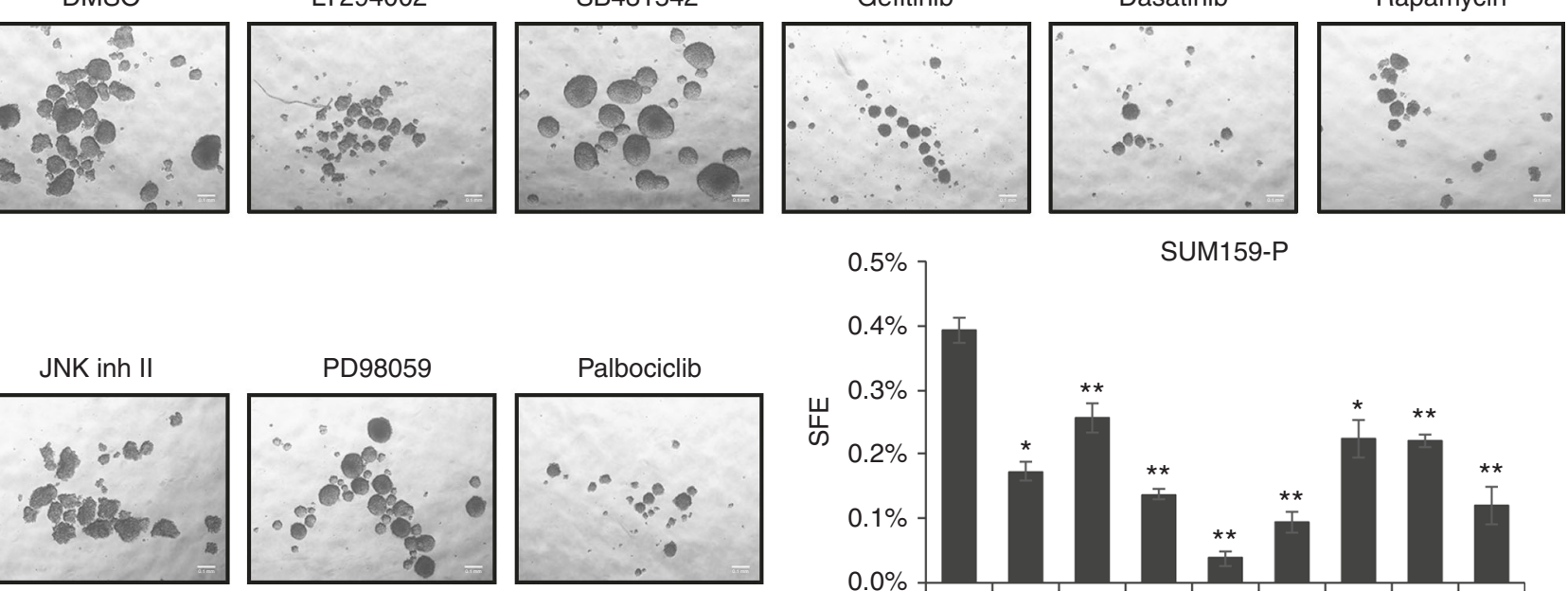

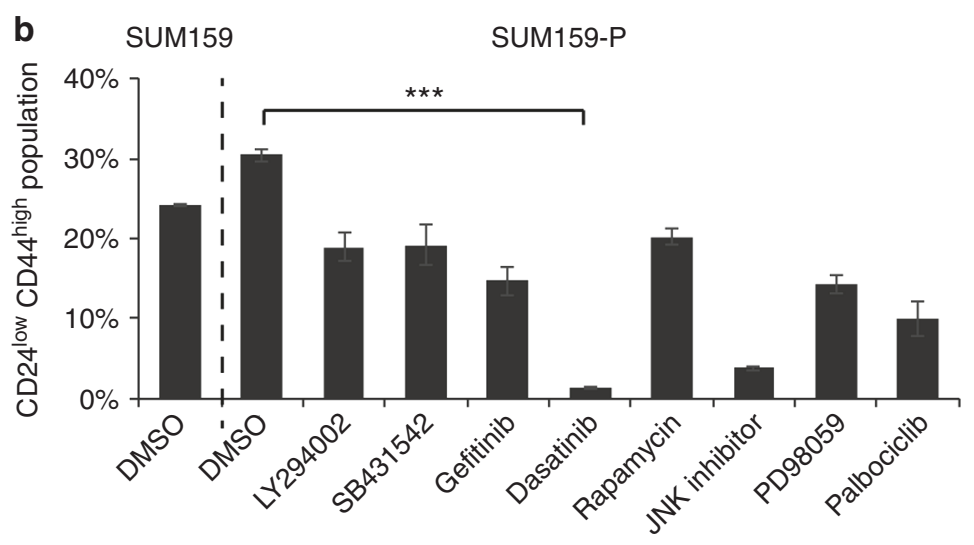

C
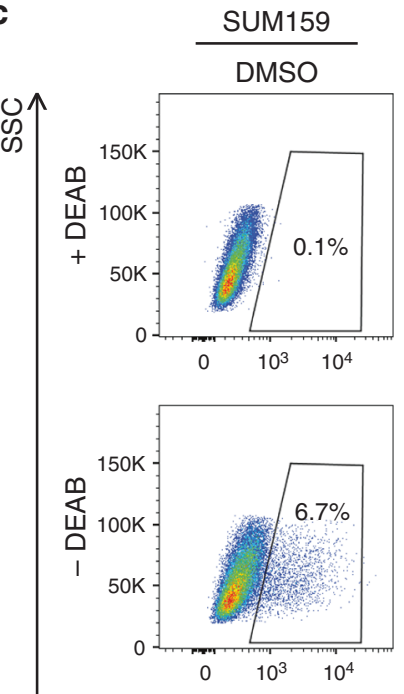
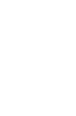
a

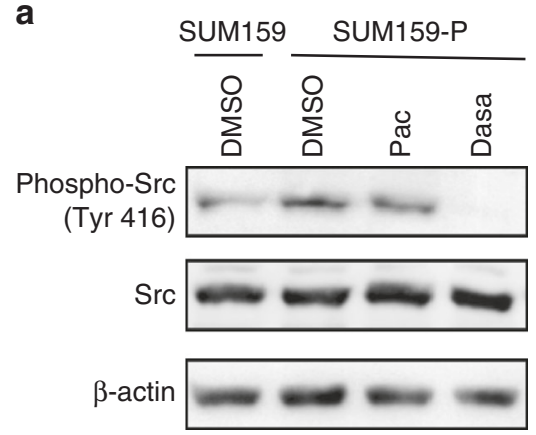

b
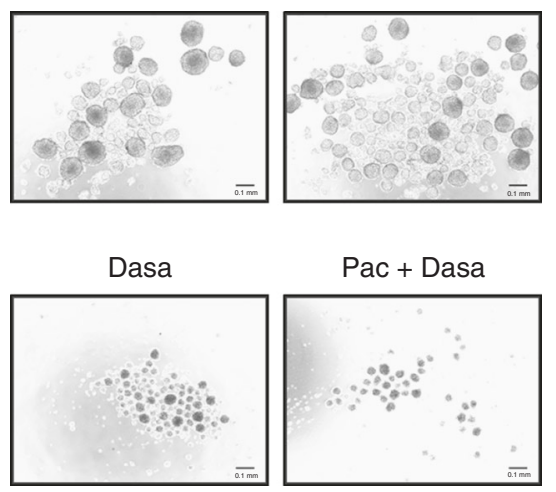

$\mathrm{Pac}+$ Dasa

SUM159

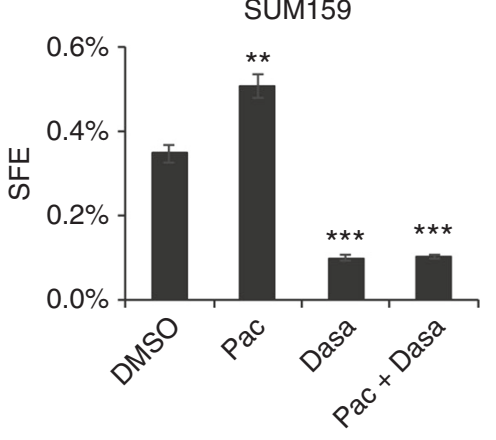

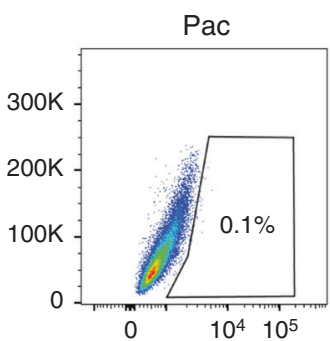

C
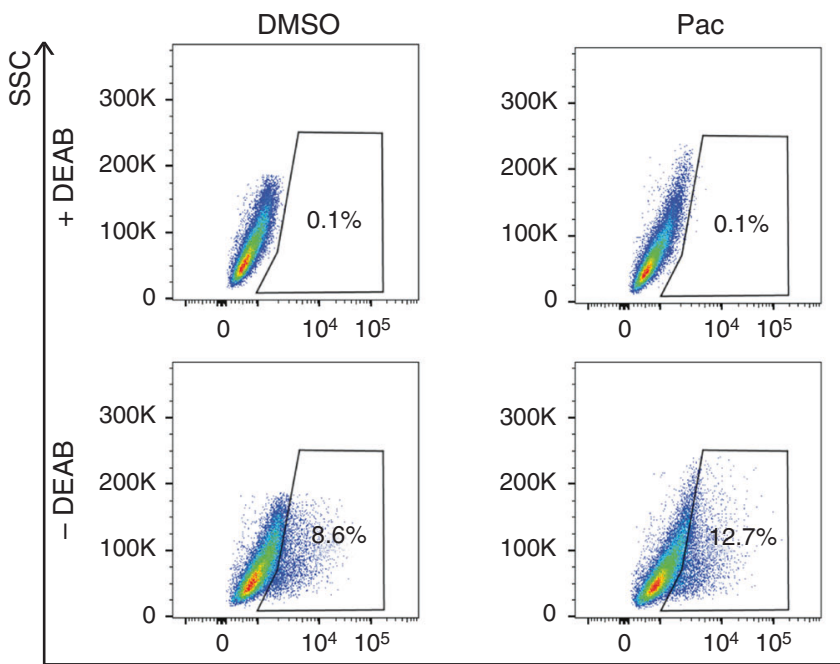
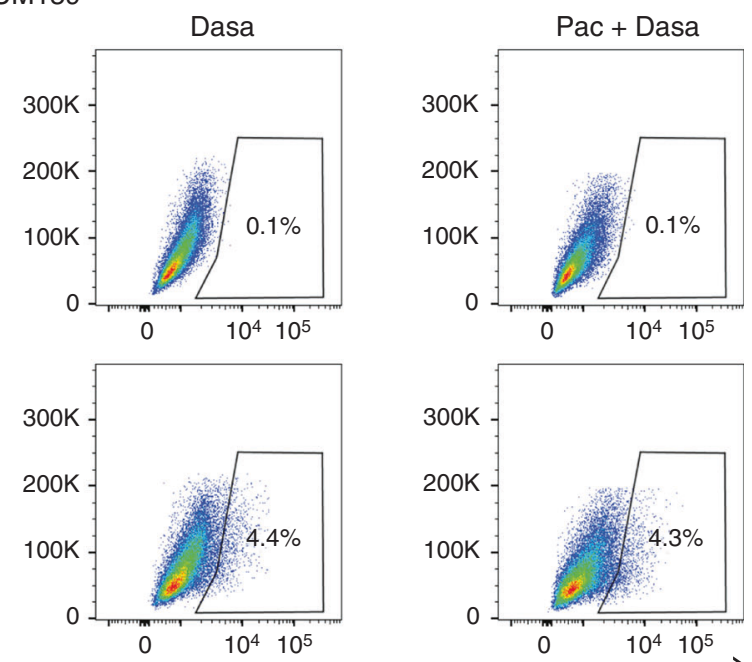

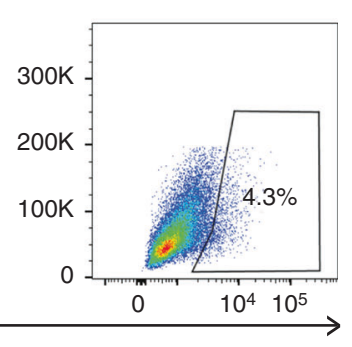

ALDH d

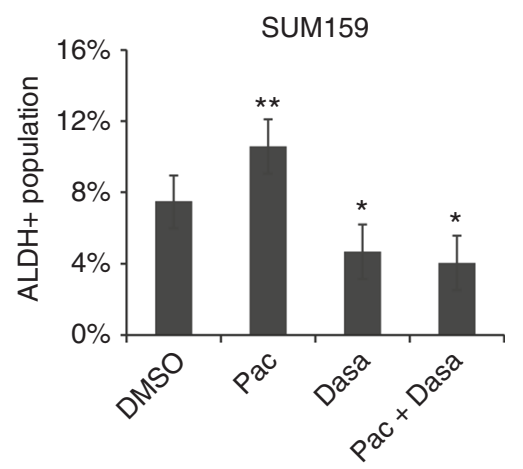

e<smiles></smiles>

Phospho-Src

(Tyr 416)

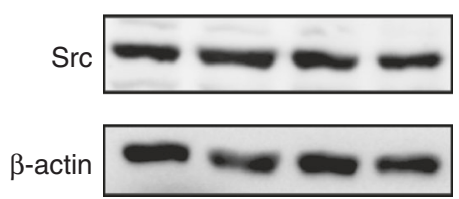

f

Fig. 3 Dasatinib suppresses BCSC through down-regulation of paclitaxel-induced Src kinase activation. a SUM159 cells treated with DMSO and SUM159-P cells treated with DMSO, paclitaxel $(10 \mathrm{nM})$, dasatinib (1 uM) were subjected with western blot analysis using anti-phospho-Src (Tyr 416), anti-Src and anti- $\beta$-actin antibodies. b Tumourspheres were derived from SUM159 cells treated with paclitaxel, dasatinib or in combination. The number of tumourspheres and SFE were determined. c, d ALDEFluor assay was conducted in SUM159 cells treated with paclitaxel, dasatinib or in combination for 4 days. The percentage of ALDH+ cells was indicated and graphed. e, f SUM159 cells were treated with paclitaxel, dasatinib or in combination for 2 days. Total cell lysates were analysed for phospho-Src (Tyr 416), Src and $\beta$-actin by western blot (e). Cell growth inhibition was measured by PrestoBlue assay (f)

proportion of both $\mathrm{CD} 24^{\text {low }} / \mathrm{CD} 44^{\text {high }}$ and ALDH+ BCSCs. Combination treatment of dasatinib/paclitaxel produced maximal reduction in $\mathrm{CD} 24^{\text {low }} / \mathrm{CD} 44^{\text {high }}$ and $\mathrm{ALDH}+\mathrm{BCSC}$ cell numbers, compared with either control-, dasatinib, or paclitaxel-treatment conditions. These results are consistent with our data using tumoursphere formation assays and further support our hypothesis that Src kinase inhibition by dasatinib sensitises BCSCs to the chemotherapy drug paclitaxel. 


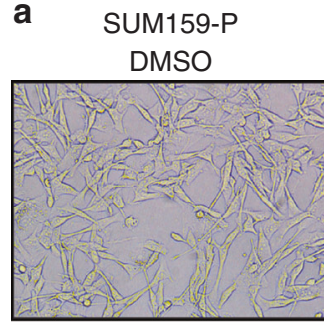

Dasatinib

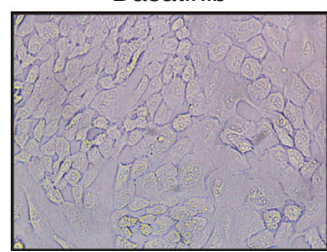

d

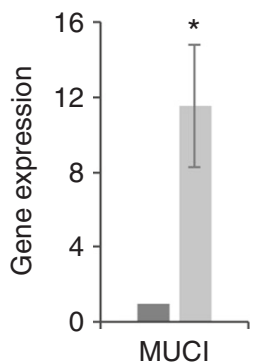

b

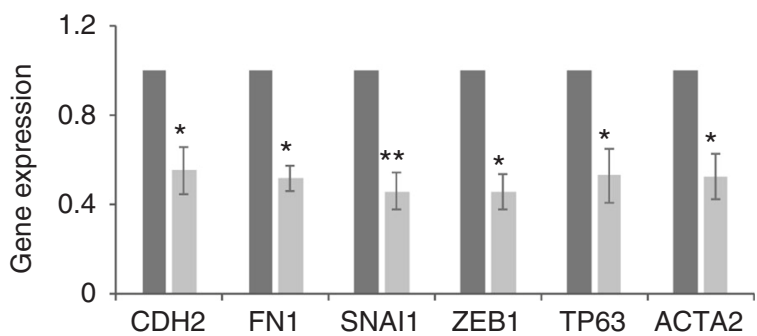

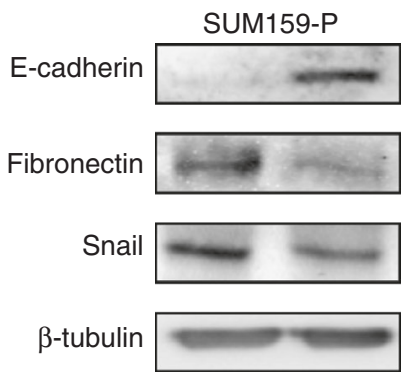

f

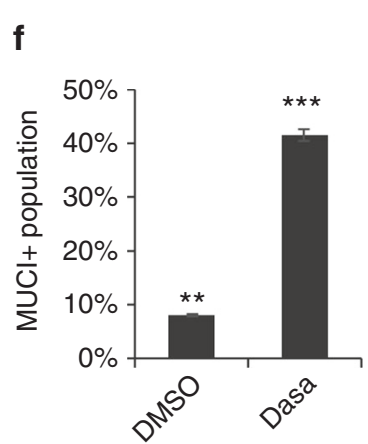

g
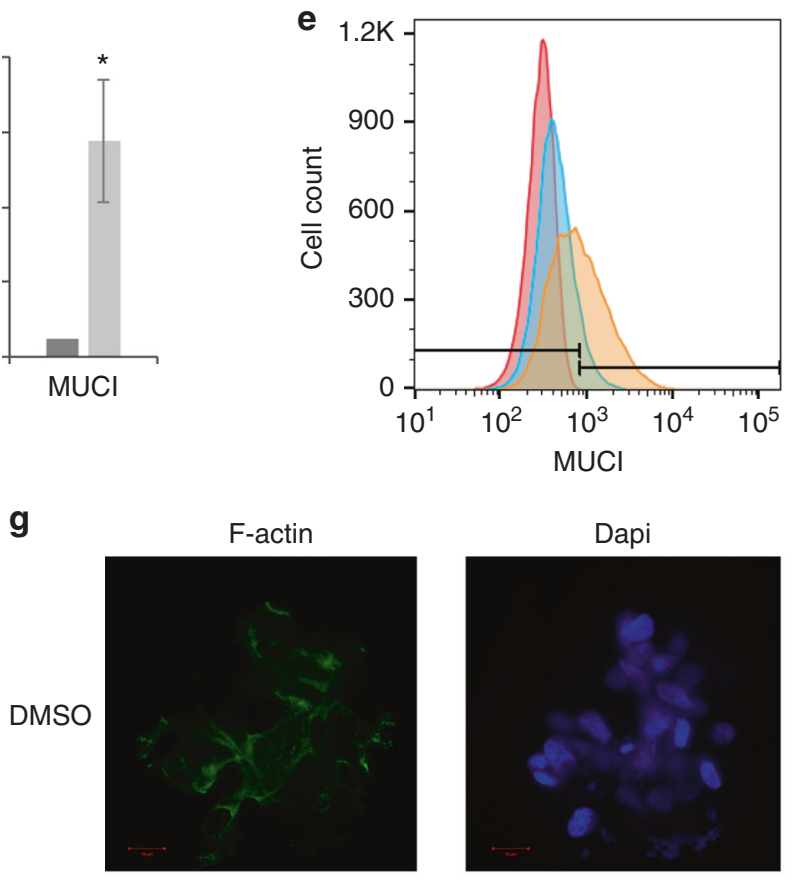

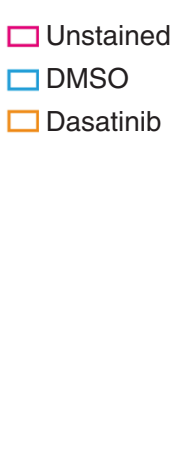

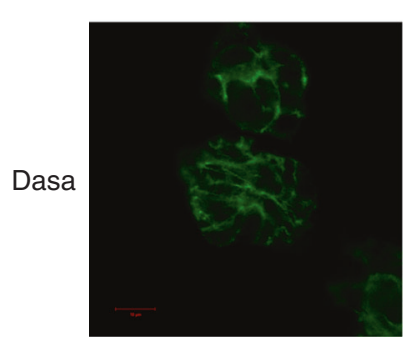
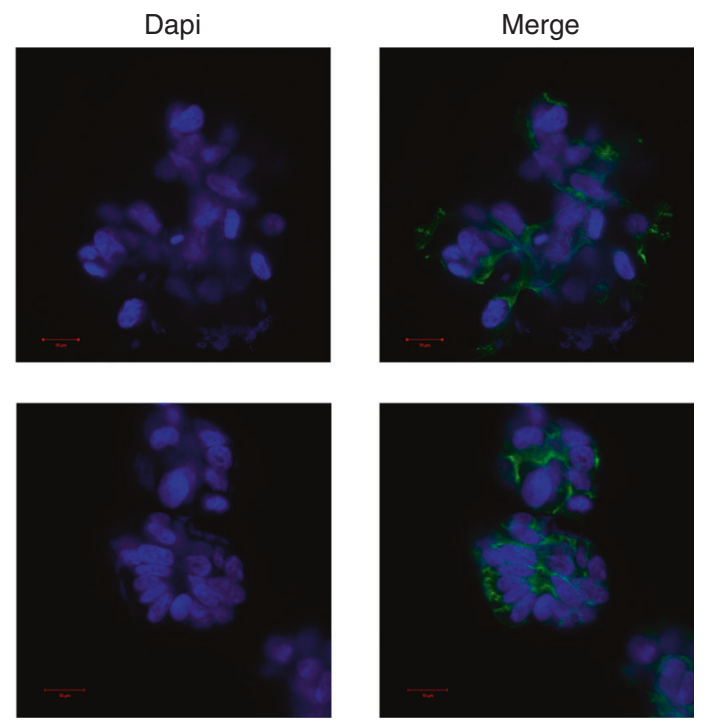

Fig. 4 Dasatinib induces an epithelial- and luminal-like phenotype and differentiation of chemo-resistant cells. a Cell morphology (20x magnification) of SUM159-P cells treated with DMSO or dasatinib (1 uM) for 4 days. b, c SUM159-P cells were treated with DMSO or dasatinib for 2 days. Then the mRNA and protein levels of indicated genes were subjected to real-time qPCR and immunoblotting analysis. $\mathbf{d}$ MUC1 mRNA expression was analysed in SUM159-P cells treated with or without dasatinib. e, f Percentage of MUC1+ population was analysed by flow cytometry in SUM159-P cells treated with dasatinib. g SUM159-P cells were treated with or without dasatinib and grown in 3D culture (Materials and Methods). Then cells were stained with antibody to F-actin (green). Nucleus was counter stained with DAPI (blue). Scale bar $=10 \mu \mathrm{m}$

Combination of dasatinib and paclitaxel potently eliminates breast cancer cells

BCSCs display resistance to conventional chemotherapy and drive tumour relapse after initial response to chemotherapy. Thus, specific eradication of BCSCs would provide an attractive solution to address the ineffectiveness of traditional breast cancer therapies. The combined use of BCSC-targeting agents with chemotherapy may target both BCSC and non-BCSC populations and offer a promising therapeutic strategy for chemotherapyresistant patients. Having shown that the combination of dasatinib 


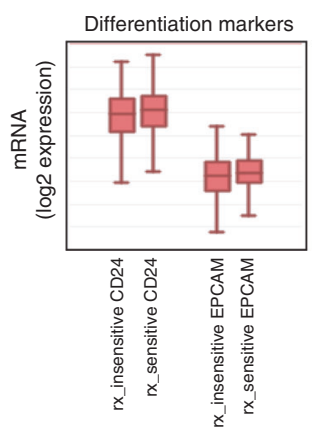

Luminal markers

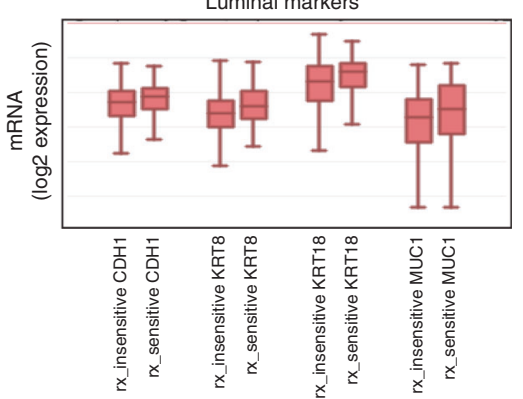

b

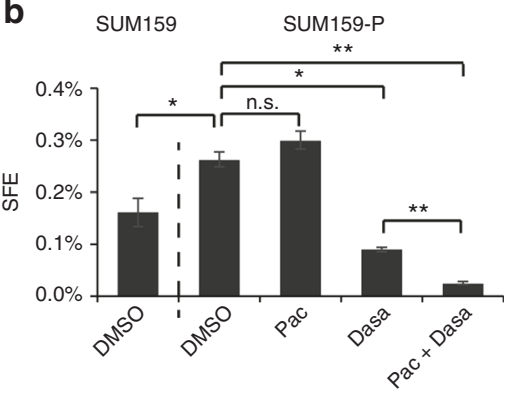

C
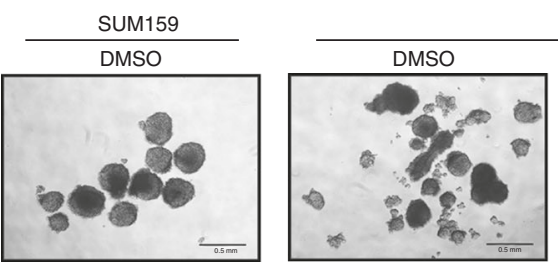

SUM159-P
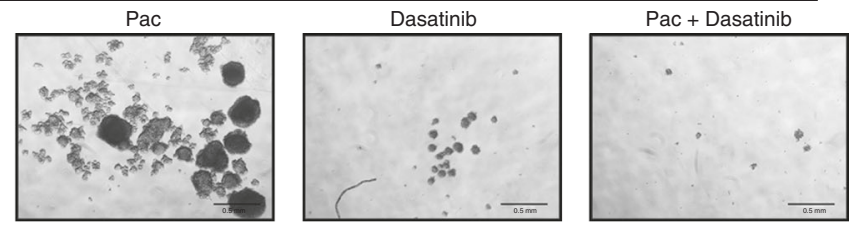

d
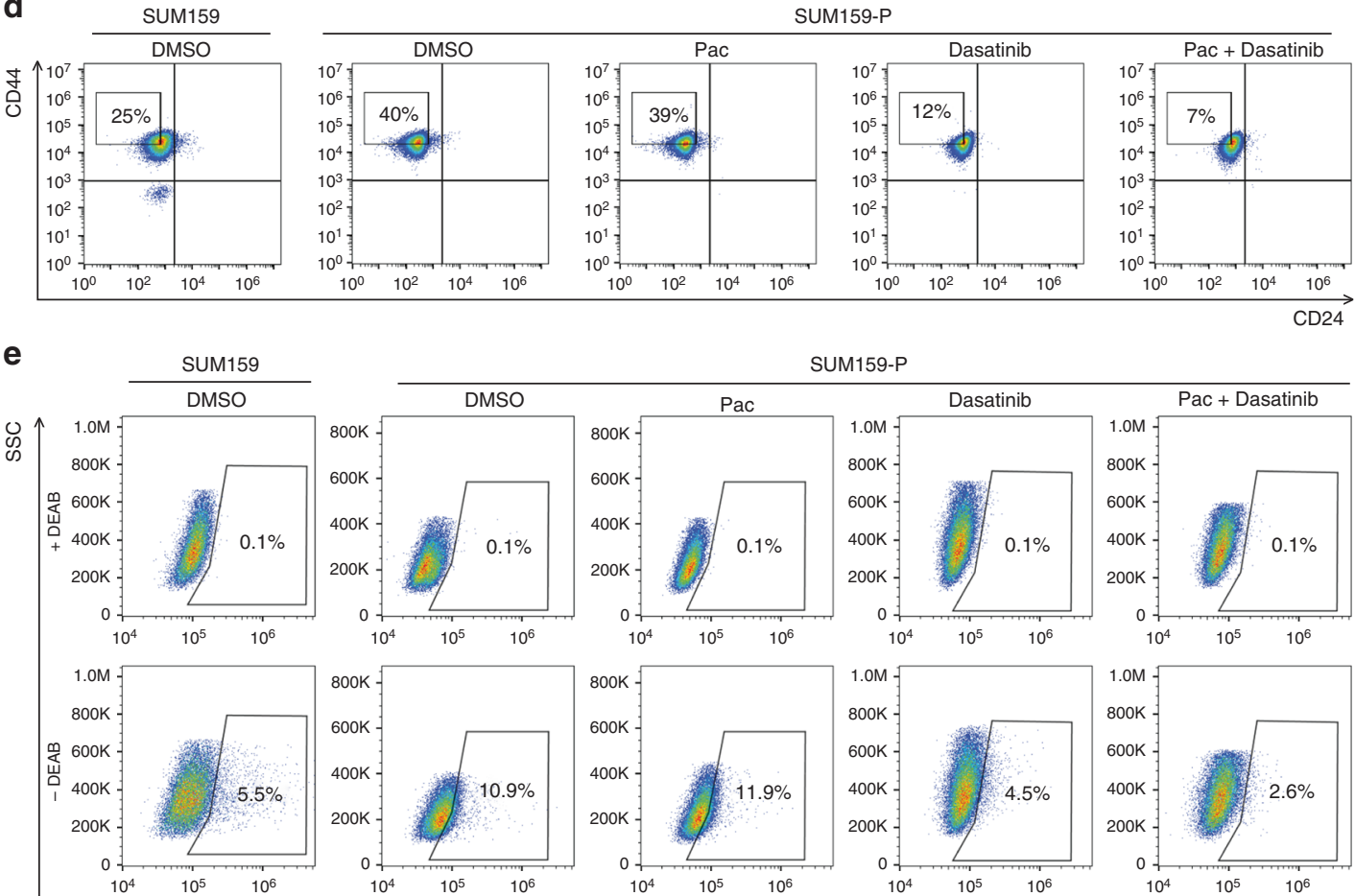

SUM159-P
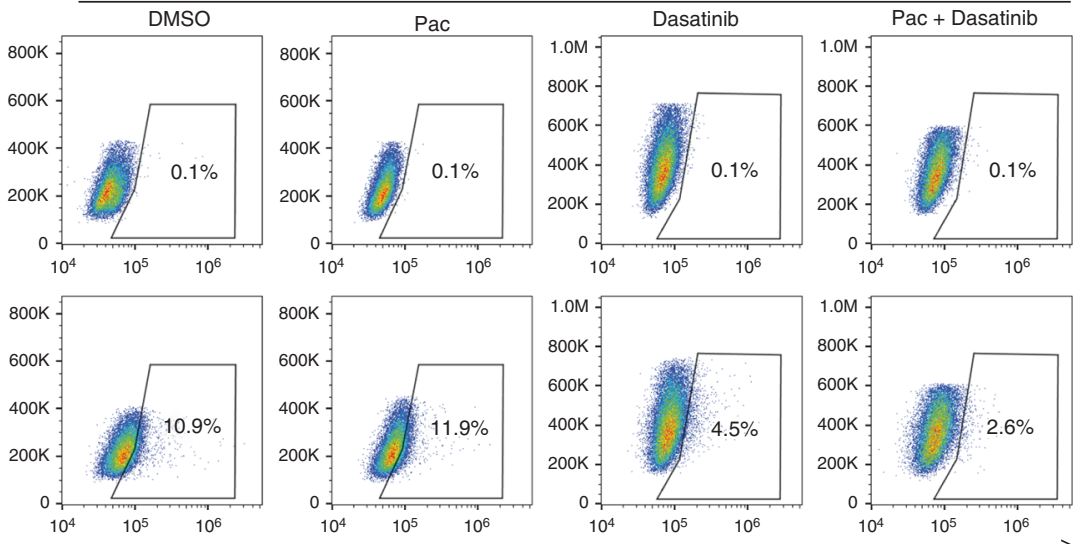

f SUM159

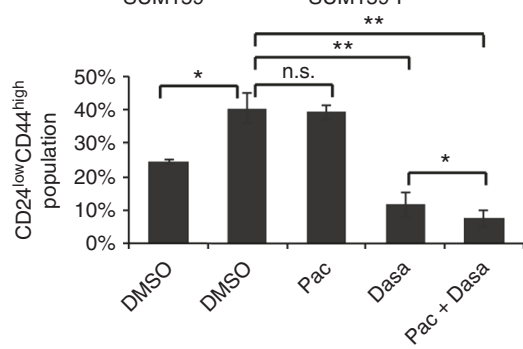

g

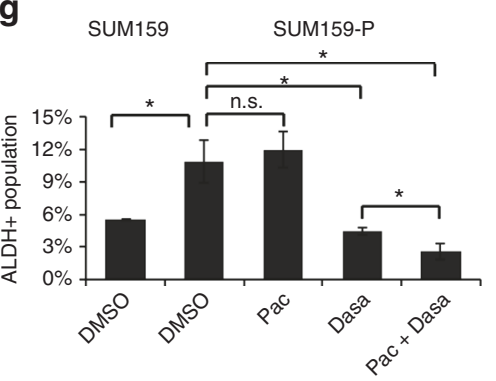

Fig. 5 Dasatinib-induced differentiation sensitises BCSCs to chemotherapy. a mRNA expression levels of the indicated genes in breast cancer patients, Booser dataset from 'R2: Genomics Analysis and Visualisation Platform (http://r2.amc.nl)'. b-g SUM159-P cells were treated with paclitaxel (10 nM), dasatinib (1 uM) or in combination. SUM159 cells were used as a control. b, c Tumoursphere formation assay was performed and the SFE was calculated. d, f Flow cytometry analysis was conducted and the percentage of CD24 ${ }^{\text {low }}$ CD44high population was assessed. e, g ALDEFluor assay was conducted and percentage of ALDH+ cells were quantified by flow cytometry 
a

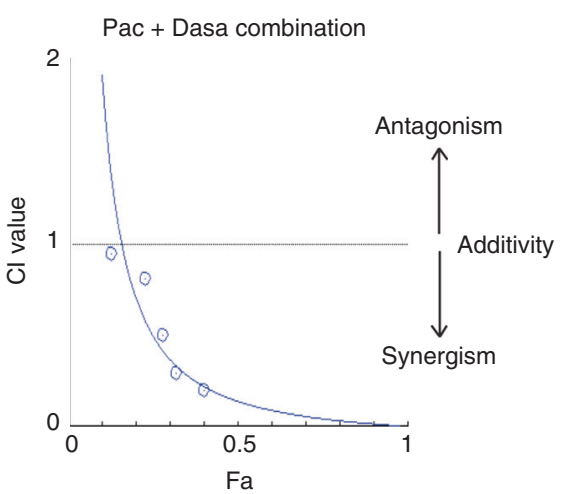

C

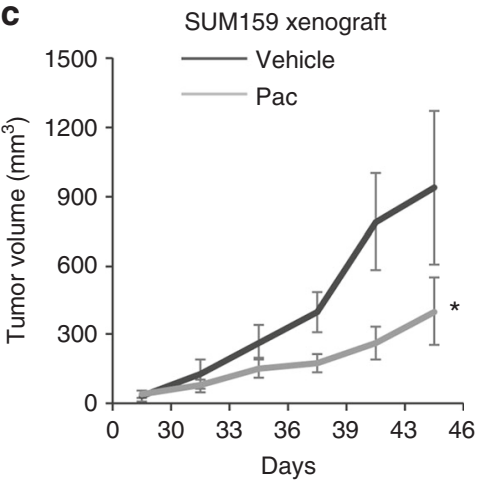

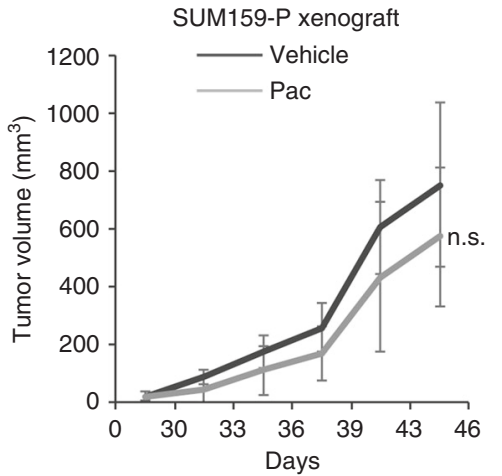

b

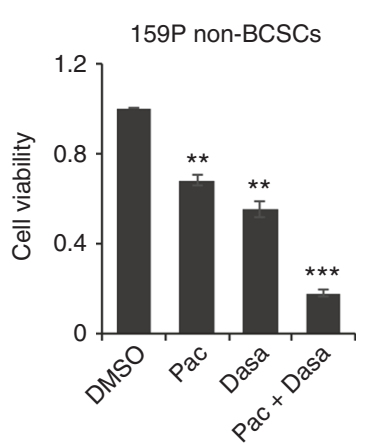

d

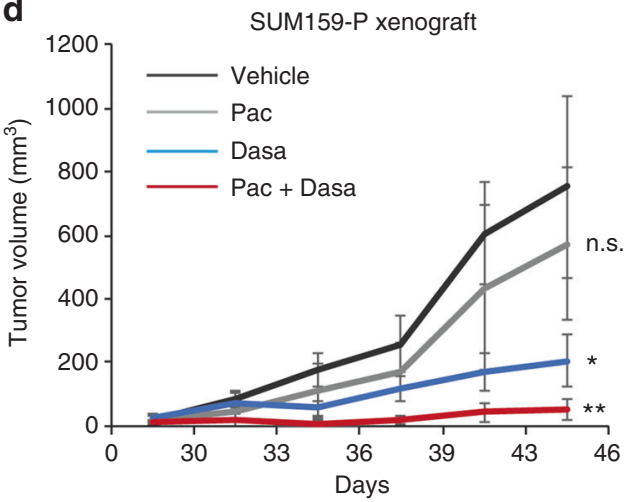

e

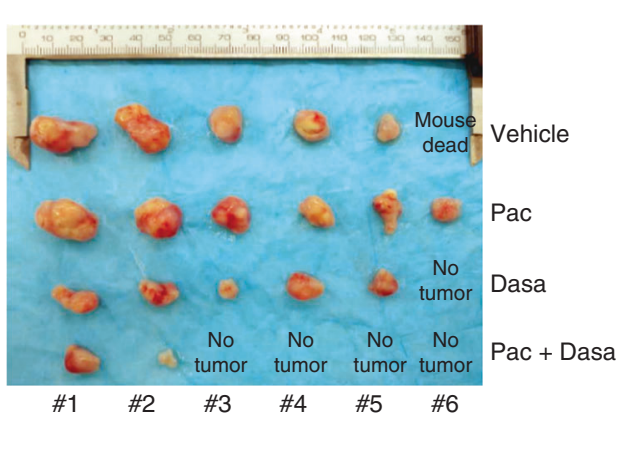

f

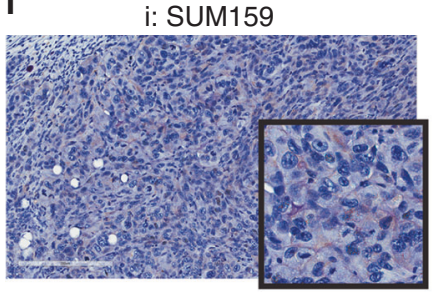

iii: SUM159-P Pac

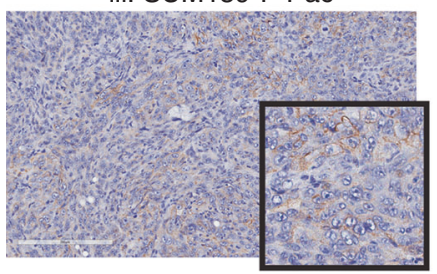

ii: SUM159-P

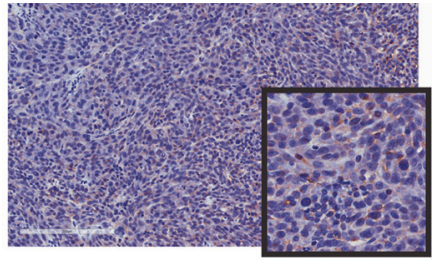

iv: SUM159-P Dasa

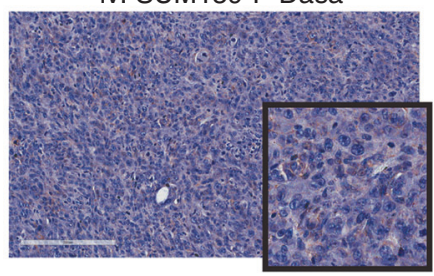

9

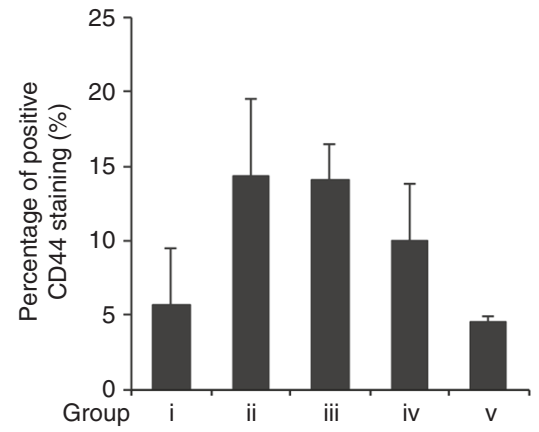

v: SUM159-P Pac+Dasa

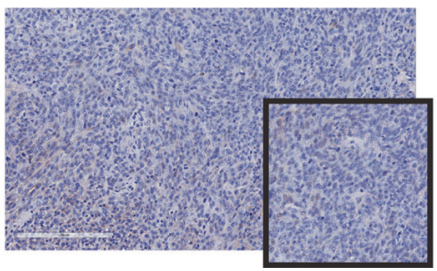

and paclitaxel decreased the fraction of chemotherapy-resistant BCSCs and their sphere forming capacity, we hypothesised that this combination treatment could enhance cytotoxicity of dasatinib and paclitaxel alone and reverse the paclitaxel resistance in SUM159-P cells. SUM159-P cells were treated with dasatinib and paclitaxel in an equi-effective fashion based on their IC50 values for 2 days and assessed for cell viability. Cytotoxicity of dasatinib/ paclitaxel combination treatment in SUM159-P was significantly higher than dasatinib or paclitaxel single-drug exposure. The $\mathrm{Cl}$-isobologram indicates that the combination of dasatinib and paclitaxel was synergistic in a wide range of fractions affected ( $\mathrm{Fa}$ ) value (Fa 0.2-Fa 0.97) (Fig. 6a). We also determined the cytotoxic effects of dasatinib, paclitaxel and combination therapy on the isolated CD24+CD44+ non-BCSCs fraction of SUM159-P and found that the concomitant dasatinib therapy enhanced the cytotoxic effect of paclitaxel in these cells (Fig. 6b). These results, 
Fig. 6 Combination of dasatinib and paclitaxel potently eliminated breast cancer cells. a Combination index (Cl)-fraction affected (Fa, corresponding to the fraction of cell viability inhibited) plot of SUM159-P cells treated with a dose range of paclitaxel in combination with dose range of dasatinib, based on a ratio of the IC50 of each drug. $\mathbf{b}$ CD24+CD44+ non-BCSCs were isolated from SUM159-P cells and subjected to PrestoBlue cell viability assay after the treatment of indicated drugs. c-e Mice were inoculated with SUM159 and SUM159-P cells and randomly grouped ( $n=6 /$ group). The treatment started as described in the Material and Methods section. c Analyses of tumour growth in SUM159 and SUM159-P derived tumours treated with or without paclitaxel. The primary tumour volume was calculated by the formula: volume $\left(\mathrm{mm}^{3}\right)=(\text { length }(\mathrm{mm}))^{2} \times($ width $(\mathrm{mm})) \times 0.5$. d Tumour growth curve of SUM159-P derived tumours treated with vehicle, paclitaxel, dasatinib or in combination. e Images of SUM159-P derived tumours in each treatment group. $\mathbf{f}, \mathbf{g}$ CD44 expression in tumour xenografts from different groups of mice. i: SUM159 xenograft. ii: SUM159-P xenograft treated with vehicle. iii: SUM159-P xenograft treated with paclitaxel alone. iv: SUM159-P xenograft treated with dasatinib alone. v: SUM159-P xenograft treated with paclitaxel and dasatinib. The score of staining intensity and the percentage of stained area were analysed. The total percentage of membrane and cytoplasmic CD44 staining was calculated for each tumour slide. $\mathbf{f}$ Representative immunohistochemistry images (20x) of CD44 in breast cancer samples. Scale bar $=200$ uM. $\mathbf{g}$ Average percentage of CD44+ area in tumour cells for each group of mice

combined with the data in Fig. 5 suggest that the combination of dasatinib and paclitaxel has a broad effect on suppressing cell survival of chemo-resistant TNBC cells, via eliminating both BCSCs and non-BCSCs.

We further examined the efficacy of dasatinib/paclitaxel combination treatment in reducing tumour volume in a preclinical model of breast cancer, using orthotopic xenografts in NSG mice. NSG mice were inoculated with SUM159 and SUM159-P cells $(1 \times$ $10^{6}$ cells per mouse) in the left and right mammary fat pad, respectively. One week following tumour cell transplantation, these mice were randomly divided into four groups (6 mice per group), and treated with vehicle, paclitaxel $(20 \mathrm{mg} / \mathrm{kg}$, once a week), dasatinib $(10 \mathrm{mg} / \mathrm{kg})$ alone or in combination for up to 6 weeks. Treatment of parental cells-derived tumours with paclitaxel efficiently reduced the tumour volume to a significant $57 \%(P<0.05)$ decrease at week 7 , compared to the vehicletreated group. As opposed, paclitaxel treatment of the SUM159-P resistant cells-derived tumours did not significantly reduce the tumour burden (Fig. 6c). Interestingly, while the dasatinib treatment suppressed SUM159-P tumour growth compared with vehicle alone $(P<0.05)$, the combination of dasatinib and paclitaxel induced near-complete tumour regression $(P<0.01)$, with four out of six mice showing no tumour at all (Fig. $6 \mathrm{~d}$ and e). Altogether, our data indicate that dasatinib significantly improves the efficacy of paclitaxel in eliminating chemo-resistant breast cancer cells both in vitro and in vivo.

Dasatinib sensitises BCSCs to paclitaxel in vivo

We next explored whether dasatinib/paclitaxel combination also led to a reduction of BCSCs in vivo. For this, we examined the stemness marker CD44 expression levels, using IHC, in tumours collected out of SUM159 and SUM159-P xenografts. As indicated in Fig. S4, both membranous and cytoplasmic staining were analysed and scored by two independent pathologists. The score of staining intensity and the percentage of area stained were indicated and the total percentage of both membranous and cytoplasmic staining was considered for further evaluation of CD44 protein expression in tumour cells. We found SUM159-Pderived tumour to display higher levels of CD44 in average $(14.3 \%$ positive) compared to that in SUM159-derived tumour $(5.7 \%$ positive) (Fig. 6f, g), demonstrating the enrichment of BCSCs in chemo-resistant breast tumour, and consistent with our in vitro findings. Although paclitaxel alone inhibited tumour growth in SUM159-P xenograft, it didn't affect the expression levels of CD44, suggesting that paclitaxel reduced tumour volume by only targeting non-BCSC populations. In contrast, we found dasatinib single treatment to decrease CD44 expression compared to vehicle-treated SUM159-P tumours. Most importantly, the dasatinib/paclitaxel combination treatment exerted even stronger effect than dasatinib alone and markedly reduced CD44 expression in SUM159-P xenograft (Fig. 6f, g). These results indicate that, by targeting BCSCs and inducing phenotypic differentiation, dasatinib can sensitise chemo-resistant BCSC towards chemotherapy in vivo.
Taken together, these data suggest the dasatinib/paclitaxel combination as a promising therapeutic to target both BCSC and non-BCSC populations and to therefore overcome chemotherapy resistance in breast cancer. Our studies provide preclinical evidence of the future combination use of dasatinib and paclitaxel to treat chemo-resistant breast cancer patients and to improve better outcomes in TNBC patients.

\section{DISCUSSION}

Triple-negative breast cancers display worse patient outcomes than any other breast cancer molecular subtypes, with significant shorter disease-free survival and overall survival times. ${ }^{40}$ Although showing an initial response to taxane- and platin-based primary chemotherapy, TNBC can develop tumour recurrences and metastases due to intrinsic and acquired chemo-resistance. The chemotherapy failure in TNBC has been partly attributed to the enrichment of BCSCs. Our study revealed that chemotherapy treatment stimulates BCSC self-renewal and expansion both in vitro and in vivo and further confers adaptive resistance, suggesting the needs to target BCSCs in chemo-resistant TNBCs. We also uncovered a novel function of Src kinase in regulating chemotherapy-induced BCSCs. Moreover, our in vitro and in vivo results highlighted that dasatinib can synergistically resensitise chemo-resistant TNBCs to paclitaxel through targeting BCSCs.

We found chemo-resistant TNBCs to exhibit increased BCSC numbers (CD24 ${ }^{\text {low }} \mathrm{CD} 44^{\text {high }}$ and $\left.\mathrm{ALDH}+\mathrm{BCSCs}\right)$ and increased sphere forming capacity. Moreover, using xenografts in NSG mice, we found chemo-resistant TNBC-derived tumours to also contain higher percentage of BCSCs. These results suggest that the longterm treatment of paclitaxel induced the expansion of BCSCs and conferred adaptive resistance to chemotherapy in TNBC. This is consistent with previous studies revealing an enriched cancer stem cell-like gene signature in neoadjuvant chemotherapytreated breast tumours ${ }^{41}$ or a link between increased BCSC percentages and intrinsic chemotherapy resistance. ${ }^{42}$ We showed that short term (4 days) exposure of TNBCs to paclitaxel also increased BCSC numbers and their tumoursphere formation ability. This suggests that short term paclitaxel treatment only kills bulk tumour cells and imposes a strong selection for drugresistant BCSC survival and expansion. Our results, combined with previous studies, emphasise the need to identify agents that efficiently eliminate BCSCs in stem-like cells enriched chemoresistant TNBC.

We also found dasatinib to efficiently reduce BCSC numbers through inhibition of pac-induced Src activation in both parental and chemo-resistant TNBCs. This is consistent with previous work showing upregulation of the stemness marker CD44 and activated Src in tumours from chemotherapy-treated mice. ${ }^{43}$ Thus, based on these observations, blocking Src activation appears as an attractive strategy to eliminate BCSCs in chemo-resistant breast cancer.

The induction of EMT in neoplastic mammary epithelial cells has been shown to enrich cells with stem-like properties. ${ }^{35}$ 
Residual breast cancers following conventional chemotherapy not only contain higher percentage of BCSCs, but also display mesenchymal features. ${ }^{41}$ Recently, researchers have been trying to identify selective inhibitors of BCSCs using high-throughput screening of compounds with EMT-specific toxicity. ${ }^{44}$ We found dasatinib treatment of chemo-resistant TNBCs to decrease BCSC numbers and induce a reversed EMT phenotype and phenotypic changes from spindle-like cell shape to cohesive clusters with round cellular morphology. Several studies have linked Src activation and EMT in pancreatic and ovarian cancer. ${ }^{45,46}$ Interestingly, a recent study suggested that activation of LYN, another dasatinib target, could in fact regulate EMT and breast cancer cell invasion. ${ }^{47}$ Thus, it will be interesting to further study the molecular mechanisms underlying these effects and further characterise the connection between dasatinib, SFK activation and EMT in breast cancer.

Recently, differentiation therapy has been shown to be an attractive way to force CSCs to differentiate terminally and therefore control tumour progression. Lombardo et al. found that bone morphogenetic protein 4 (BMP4), which promotes terminal differentiation of colorectal cancer stem cells via inhibition of Wnt signalling, could increase their sensitivity to chemotherapy. ${ }^{7}$ Another study showed that all-trans-retinoic acid treatment induced BCSC differentiation, leading to a shrinkage of the BCSC population. ${ }^{48}$ We show that dasatinib treatment leads to the formation of acinar-like structure of chemo-resistant TNBCs in 3D Matrigel culture and to the increased expression of luminal differentiation markers, indicating that dasatinib could induce a phenotypic differentiation of BCSC-enriched chemo-resistant cells. Thus, dasatinib could prove useful to induce phenotypic differentiation of breast cancer stem cells.

It has been established that differentiated cancer cells are more sensitive to chemotherapy. In this study, we found that dasatinib treatment not only induced epithelial differentiation of BCSC-enriched chemo-resistant cells but also sensitised these cells to paclitaxel. We further found that dasatinib and paclitaxel had a synergistic effect on reducing cell viability of chemoresistant cells in vitro and in reducing tumour growth in vivo. This is consistent with findings from clinical trials, where single dasatinib treatment did not exhibit significant antitumour activity in patients with metastatic breast cancer, ${ }^{49,50}$ while a recent phase I clinical trial in metastatic breast cancer (BristolMyers Squibb Study CA180194) showed that dasatinib plus weekly paclitaxel treatment proved more effective and produced a partial response in 4 of 13 patients (31\%), including two patients previously treated with taxanes. ${ }^{51}$ Thus, our study has several translational implications. First, it reveals that chemotherapy treatment triggers BCSC sphere forming capacity and expansion and further confers adaptive resistance, highlighting the needs to target BCSCs in chemo-resistant TNBCs. Secondly, we uncovered a new role for Src kinase in mediating chemotherapyinduced BCSC properties and found dasatinib as a potent blocker of cancer stemness, further highlighting the potential clinical value of the SFK inhibitor, dasatinib as an efficient anti-stemness cancer drug in TNBCs. Thirdly, although dasatinib is well-tolerated clinically applicable Src inhibitor, the results from phase II clinical trials of dasatinib monotherapy on solid tumours are discouraging. ${ }^{52}$ One possible explanation for this issue in breast cancer was that patients were unselected in the clinical trials of dasatinib. Given the role of Src kinase in paclitaxel resistant BC, the activation of Src kinase may serve as a response biomarker for the dasatinib and paclitaxel combination treatment in TNBCs. Last, our in vitro and in vivo results indicate that dasatinib can resensitise refractory breast cancer cells to paclitaxel. Although a similar trend was observed in the phase I clinical trial (BristolMyers Squibb Study CA180194) in metastatic breast cancer, ${ }^{51}$ the underlying mechanisms remained unclear. We show here that dasatinib, in fact induces epithelial differentiation of chemo-resistant TNBCs and that combination of dasatinib and paclitaxel led to the near-complete elimination of BCSCs. Thus, our data could explain the observed results from the Bristol-Myers Squibb phase I trial. In the future, it will be interesting to also investigate the dasatinib involvement in other BCSC-related chemo-resistance mechanisms, including the expression of drug export proteins, induction of quiescence, activation of stemness signalling pathway.

Our study highlights the potential clinical utility of dasatinib/ paclitaxel combinatorial treatment to overcome chemotherapy resistance and to improve better outcomes in TNBC patients. Several previous studies also tested the use of dasatinib in combination with other drugs as potential therapeutics in different types of breast cancer. For instance, combination of Src inhibitor and anti-oestrogen therapy effectively stimulated cancer cell cycle arrest and suppressed Src inhibitor resistance in ER+ breast cancer. ${ }^{53}$ Moreover, for HER2 overexpressing breast cancer, although Src inhibitor alone did not show effects in treating trastuzumab or lapatinib-resistant tumours, targeting Src signalling pathway significantly sensitised these resistant tumours to anti-HER2 treatments. ${ }^{54}$ These studies, combined with our present findings in TNBCs highlight the potential benefit of using SrC inhibitor, especially in combinatorial regimens for treating different types of breast cancers.

\section{ACKNOWLEDGEMENTS}

The authors thank Dr. Stephen Ethier for help with providing SUM159PT cell line. We would also like to thank Aida Kasei for her help with the animal work.

\section{AUTHOR CONTRIBUTIONS}

J.T. designed, performed experiments, analysed data and drafted the manuscript. F.A.R. was involved in performing PCR and western blot experiments. M.D. contributed to designing and performing experiments. A.M. assisted in 3D cell culture. B.K., I.Y.H. and K.B. were involved in IHC analysis. S.A. and B.J. assisted in designing experiments and editing the manuscript. J.J.L. was involved in research design, data interpretation, supervision of the project and drafting the manuscript.

\section{ADDITIONAL INFORMATION}

Supplementary Information is available for this paper at https://doi.org/10.1038/ s41416-018-0287-3.

Competing interests: The authors declare no competing interests.

Ethics approval and consent to participate: The study was performed in accordance with the Declaration of Helsinki.

Funding: This study was funded by grant from the Canadian Institutes for Health Research (CIHR to J.J.L.).

Note: This work is published under the standard license to publish agreement. After 12 months the work will become freely available and the license terms will switch to a Creative Commons Attribution-NonCommercial-Share Alike 4.0 Unported License.

\section{REFERENCES}

1. Schneider, B. P. et al. Triple-negative breast cancer: risk factors to potential targets. Clin. Cancer Res. 14, 8010-8018 (2008).

2. Andre, F. \& Zielinski, C. C. Optimal strategies for the treatment of metastatic triple-negative breast cancer with currently approved agents. Ann. Oncol. 23, vi46-51 (2012).

3. Magee, J. A., Piskounova, E. \& Morrison, S. J. Cancer stem cells: impact, heterogeneity, and uncertainty. Cancer Cell 21, 283-296 (2012).

4. Wicha, M. S., Liu, S. \& Dontu, G. Cancer stem cells: an old idea-a paradigm shift. Cancer Res. 66, 1883-1890 (2006).

5. Al-Hajj, M., Wicha, M. S., Benito-Hernandez, A., Morrison, S. J. \& Clarke, M. F. Prospective identification of tumorigenic breast cancer cells. Proc. Natl Acad. Sci. USA 100, 3983-3988 (2003). 
6. Ginestier, C. et al. ALDH1 is a marker of normal and malignant human mammary stem cells and a predictor of poor clinical outcome. Cell Stem Cell 1, 555-567 (2007).

7. Lombardo, Y. et al. Bone morphogenetic protein 4 induces differentiation of colorectal cancer stem cells and increases their response to chemotherapy in mice. Gastroenterology 140, 297-309 (2011).

8. Wielenga, M. C. B. et al. ER-stress-induced differentiation sensitizes colon cancer stem cells to chemotherapy. Cell Rep. 13, 489-494 (2015).

9. Campos, B. et al. Differentiation therapy exerts antitumor effects on stem-like glioma cells. Clin. Cancer Res. 16, 2715-2728 (2010).

10. Yeatman, T. J. A renaissance for SRC. Nat. Rev. Cancer 4, 470-480 (2004).

11. Wheeler, D. L., lida, M. \& Dunn, E. F. The role of Src in solid tumors. Oncologist 14, 667-678 (2009)

12. Ahluwalia, M. S., de Groot, J., Liu, W. M. \& Gladson, C. L. Targeting SRC in glioblastoma tumors and brain metastases: rationale and preclinical studies. Cancer Lett. 298, 139-149 (2010).

13. Summy, J. M. \& Gallick, G. E. Src family kinases in tumor progression and metastasis. Cancer Metastas-. Rev. 22, 337-358 (2003).

14. Zhang, X. H. et al. Latent bone metastasis in breast cancer tied to Src-dependent survival signals. Cancer Cell 16, 67-78 (2009).

15. Thakur, R., Trivedi, R., Rastogi, N., Singh, M. \& Mishra, D. P. Inhibition of STAT3, FAK and Src mediated signaling reduces cancer stem cell load, tumorigenic potential and metastasis in breast cancer. Sci. Rep. 5, 10194 (2015).

16. Steinberg, M. Dasatinib: a tyrosine kinase inhibitor for the treatment of chronic myelogenous leukemia and philadelphia chromosome-positive acute lymphoblastic leukemia. Clin. Ther. 29, 2289-2308 (2007).

17. Lehmann, B. D. et al. Identification of human triple-negative breast cancer subtypes and preclinical models for selection of targeted therapies. J. Clin. Invest. 121, 2750-2767 (2011)

18. Kurebayashi, J. et al. Preferential antitumor effect of the Src inhibitor dasatinib associated with a decreased proportion of aldehyde dehydrogenase 1-positive cells in breast cancer cells of the basal B subtype. BMC Cancer 10, 568 (2010).

19. Tian, J. et al. Cyclooxygenase- 2 regulates TGFbeta-induced cancer stemness in triple-negative breast cancer. Sci. Rep. 7, 40258 (2017).

20. Dai, M. et al. CDK4 regulates cancer stemness and is a novel therapeutic target for triple-negative breast cancer. Sci. Rep. 6, 35383 (2016).

21. Charafe-Jauffret, E. et al. Breast cancer cell lines contain functional cancer stem cells with metastatic capacity and a distinct molecular signature. Cancer Res. 69, 1302-1313 (2009).

22. Fillmore, C. M. \& Kuperwasser, C. Human breast cancer cell lines contain stem-like cells that self-renew, give rise to phenotypically diverse progeny and survive chemotherapy. Breast Cancer Res. 10, R25 (2008).

23. Bhola, N. E. et al. TGF-beta inhibition enhances chemotherapy action against triple-negative breast cancer. J. Clin. Invest. 123, 1348-1358 (2013).

24. Ponti, D. et al. Isolation and in vitro propagation of tumorigenic breast cancer cells with stem/progenitor cell properties. Cancer Res. 65, 5506-5511 (2005).

25. lorns, E. et al. A new mouse model for the study of human breast cancer metastasis. PLoS ONE 7, e47995 (2012).

26. Massihnia, D. et al. Triple negative breast cancer: shedding light onto the role of pi3k/akt/mtor pathway. Oncotarget 7, 60712-60722 (2016)

27. Lebrun, J.-J. The dual role of TGF $\beta$ in human cancer: from tumor suppression to cancer metastasis. ISRN Mol. Biol. 2012, 28 (2012).

28. Crown, J., O'Shaughnessy, J. \& Gullo, G. Emerging targeted therapies in triplenegative breast cancer. Ann. Oncol. 23, vi56-65 (2012).

29. Kalimutho, M. et al. Targeted therapies for triple-negative breast cancer: combating a stubborn disease. Trends Pharmacol. Sci. 36, 822-846 (2015).

30. Xie, X. et al. c-Jun N-terminal kinase promotes stem cell phenotype in triplenegative breast cancer through upregulation of Notch1 via activation of c-Jun. Oncogene 36, 2599-2608 (2017).
31. Xia, P. \& Xu, X. Y. PI3K/Akt/mTOR signaling pathway in cancer stem cells: from basic research to clinical application. Am. J. Cancer Res. 5, 1602-1609 (2015).

32. Cheng, J. et al. MEK1 signaling promotes self-renewal and tumorigenicity of liver cancer stem cells via maintaining SIRT1 protein stabilization. Oncotarget 7, 20597-20611 (2016).

33. Chen, F. JNK-induced apoptosis, compensatory growth, and cancer stem cells. Cancer Res. 72, 379-386 (2012).

34. Abhold, E. L. et al. EGFR kinase promotes acquisition of stem cell-like properties: a potential therapeutic target in head and neck squamous cell carcinoma stem cells. PLOS ONE 7, e32459 (2012).

35. Mani, S. A. et al. The epithelial-mesenchymal transition generates cells with properties of stem cells. Cell 133, 704-715 (2008).

36. Edmondson, R., Broglie, J. J., Adcock, A. F. \& Yang, L. Three-dimensional cell culture systems and their applications in drug discovery and cell-based biosensors. Assay. Drug Dev. Technol. 12, 207-218 (2014).

37. Liu, F. et al. Prolactin/Jak2 directs apical/basal polarization and luminal linage maturation of mammary epithelial cells through regulation of the Erk1/2 pathway. Stem Cell Res. 15, 376-383 (2015).

38. Dimri, M. et al. Modeling breast cancer-associated c-Src and EGFR overexpression in human MECs: c-Src and EGFR cooperatively promote aberrant threedimensional acinar structure and invasive behavior. Cancer Res 67, 4164-4172 (2007).

39. Harma, V. et al. A comprehensive panel of three-dimensional models for studies of prostate cancer growth, invasion and drug responses. PLoS ONE 5, e10431 (2010)

40. Dent, R. et al. Triple-negative breast cancer: clinical features and patterns of recurrence. Clin. Cancer Res. 13, 4429-4434 (2007).

41. Creighton, C. J. et al. Residual breast cancers after conventional therapy display mesenchymal as well as tumor-initiating features. Proc. Natl Acad. Sci. USA 106, 13820-13825 (2009).

42. $\mathrm{Li}, \mathrm{X}$. et al. Intrinsic resistance of tumorigenic breast cancer cells to chemotherapy. J. Natl. Cancer Inst. 100, 672-679 (2008).

43. Goldman, A. et al. Temporally sequenced anticancer drugs overcome adaptive resistance by targeting a vulnerable chemotherapy-induced phenotypic transition. Nat. Commun. 6, 6139 (2015).

44. Gupta, P. B. et al. Identification of selective inhibitors of cancer stem cells by highthroughput screening. Cell 138, 645-659 (2009).

45. Nagathihalli, N. S. \& Merchant, N. B. Src-mediated regulation of E-cadherin and EMT in pancreatic cancer. Front Biosci. (Landmark Ed.). 17, 2059-2069 (2012).

46. Fang, D. et al. Epithelial-mesenchymal transition of ovarian cancer cells is sustained by Rac1 through simultaneous activation of MEK $1 / 2$ and Src signaling pathways. Oncogene 36, 1546-1558 (2017).

47. Choi, Y. L. et al. LYN is a mediator of epithelial-mesenchymal transition and a target of dasatinib in breast cancer. Cancer Res. 70, 2296-2306 (2010).

48. Ginestier, $C$. et al. Retinoid signaling regulates breast cancer stem cell differentiation. Cell Cycle 8, 3297-3302 (2009).

49. Herold, C. I. et al. Phase II trial of dasatinib in patients with metastatic breast cancer using real-time pharmacodynamic tissue biomarkers of Src inhibition to escalate dosing. Clin. Cancer Res. 17, 6061-6070 (2011).

50. Finn, R. S. et al. Dasatinib as a single agent in triple-negative breast cancer: results of an open-label phase 2 study. Clin. Cancer Res. 17, 6905-6913 (2011).

51. Fornier, M. N. et al. A phase I study of dasatinib and weekly paclitaxel for metastatic breast cancer. Ann. Oncol. 22, 2575-2581 (2011).

52. Mayer, E. L. \& Krop, I. E. Advances in targeting SRC in the treatment of breast cancer and other solid malignancies. Clin. Cancer Res. 16, 3526-3532 (2010).

53. Chen, Y. et al. Combined Src and ER blockade impairs human breast cancer proliferation in vitro and in vivo. Breast Cancer Res. Treat. 128, 69-78 (2011).

54. Zhang, S. et al. Combating trastuzumab resistance by targeting SRC, a common node downstream of multiple resistance pathways. Nat. Med. 17, 461-469 (2011). 